\title{
XIII IMBG All-Ukrainian Conference of Young Scientists
}

\section{Hemagglutinin antigenic sites changes of pandemic influenza viruses A(H1N1)PDM09 isolated in 2009-2017 in Ukraine}

\author{
O. Zolotarova ${ }^{1,2}$, L. V. Radchenko², L. V. Leibenko², I. G. Budzanivska ${ }^{1}$, A. P. Mironenko ${ }^{2}$ \\ ${ }^{1}$ ESC "Institute of Biology and Medicine", Taras Shevchenko National University of Kyiv, \\ Kyiv, Ukraine \\ ${ }^{2}$ SI "Gromashevsky L.V. Institute of epidemiology and infectious diseases, NAMS of Ukraine", \\ Kyiv, Ukraine
}

Background. The influenza A(H1N1)pdm2009 (H1N1/2009) virus, that emerged during March and early April 2009, spread rapidly among humans to develop into the first human influenza pandemic over 40 years. Hemagglutinin is known to be a major target region of neutralizing antibodies, which inhibit binding with sialic acid receptors effectively. The virus evades these antibodies primarily by accumulating amino-acid substitutions in the HA's antigenic sites. It is known that the $\mathrm{H} 1$ molecule has five antigenic sites: $\mathrm{Sa}, \mathrm{Sb}, \mathrm{Ca} 1, \mathrm{Ca} 2$, and $\mathrm{Cb}$. The antigenic sites $\mathrm{Sa}$ and $\mathrm{Sb}$, which contain the largest number of amino acid residues, are key in neutralizing epitopes that are adjacent to the receptor-binding pocket. The aim of our work was to analyze variability of [the] influenza viruses A(H1N1)pdm09 amino acid substitutions in antigenic sites of hemagglutinin. Methods. Nasal-throat swabs taken from influenza-affected patients from different regions of Ukraine, collected during 2009-2017, were used in the study. The samples were analyzed using real-time polymerase chain reaction (RT-PCR). Influenza viruses were isolated in MDCK and MDCK-SIAT cell culture. Sequencing of influenza viruses genes, isolated in our laboratory, was performed in the World Influenza Center in London using the technology of RNA-SEQ, which allows sequencing coding and noncoding mRNA. [The] Nucleotide sequences were translated into [the] amino acid sequences using MEGA 6 software. Results. Ukrainian isolates between 2009-2017 years clustered in the influenza genetic groups 2, 6, 7, and 8. Genetic changes were observed in each of the antigenic sites: Sa - S162T, K163Q, K163I; Sb - S185T, A186T, S190G, S190R; Ca1 - S203T, R205K, E235V, E235D, S236P; Ca2 - P137H, H138R, A141T, D222G, D222N; Cb - A73S, S74R, $\mathrm{S} 74 \mathrm{~N}$. The greatest number was detected on the sites $\mathrm{Ca} 1$ and $\mathrm{Ca} 2$. The smallest number of amino acid substitutions was detected in the antigenic site $\mathrm{Cb}$. In spite of detected mutations in antigenic sites, Ukrainian isolates retained the similarity to the vaccine strain $\mathrm{A} / \mathrm{California} / 07 / 09$ during 2009-2017. Conclusions. Information about the changes in antigenic sites is very important for prediction of the next dominant strains. It is well-documented that antigenic changes of HA occasionally result in the acquisition of carbohydrate side chains on the HA molecule. Since the carbohydrate side chains in the vicinity of antigenic sites mask the neutralizing epitopes on the HA surface, the amino acid substitutions associated with the acquisition of carbohydrate chains are believed to efficiently generate antigenic variants. 


\title{
Screening of gene-partners for swiss cheese GENE of Drosophila Melanogaster
}

\author{
M. Horin ${ }^{1}$, N. Matiytsiv ${ }^{1}$ \\ ${ }^{1}$ Ivan Franko National University of Lviv, \\ Lviv, Ukraine, Faculty of Biology, Department of Genetics and Biotechnology
}

Background. Swiss cheese (sws) is a Drosophila melanogaster ortholog of human Neuropathy target esterase (NTE or PLPLA6), a molecular target for the organophosphorus compoundinduced delayed neuropathy (OPIDN). SWS is a transmembrane protein, loss of its function causes age-dependent neurodegeneration, glial hyperwrapping, and neuronal apoptosis. As shown previously, the sws mutants have decreased lifespan. This phenotype is accelerated even further when animals are starved or fed with organophosphates. Our aim was to find genes-partners of sws in order to clarify in which cell the signaling SWS is involved and to understand better the cellular mechanism of SWS/NTE-dependent neuropathy formation. Methods. Based on in-silico predictions and theoretical searching we selected 25 candidate genes that can potentially interact with SWS. To verify their functional interactions with SWS in vivo, we analyzed heterozygous animals with a reduction by one copy of the candidate gene in the sws mutant background (sws1). We analyzed three sws-related phenotypes: sensitivity to starvation, viability after organophosphate Diazinon treatment and brain tissue degeneration. To verify the sensitivity to starvation, 4-6 day-old flies were collected and transferred to vials with [the] control solution (10\% sucrose, $1 \%$ yeast extract) or only with water; during next 6 days we counted dead flies. After 24-hour exposition to $0.0015 \mathrm{mg} / \mathrm{ml}$ Diazinon[, the] flies were transferred to a standard food vial, and their survival during next 14 days was estimated using GraphPad Prism 6 (Graphpad Software Inc., La Jolla, CA, USA). Log-rank test was used to estimate statistically significant difference. For brain tissue analysis we made paraffin sections of head from 20 day-old flies. Histological preparations were analyzed in UV light using a microscope Loboval-3 (Zeiss-Jena, Germany). Results. We tested if downregulation of a potential interactor would rescue or further accelerate the reduced lifespan phenotype under stress conditions. Currently, we have tested 11 out of 25 candidate genes. Our analysis showed that several genes: Dl (Delta), rl (rolled), ena (enabled), nes (nessy), Lar (Leukocyteantigen-related-like) and park show genetic interactions with sws. Interestingly, heterozygous flies for Dl, rl, nes, Lar and ena were resistant to both stress factors - Diazinon treatment and nutrition deprivation (starvation) and could partially rescue sws phenotype. Reduction of $\mathrm{Dl}$, ena and $\mathrm{rl}$ in the sws mutants could also rescue severe brain neurodegeneration observed in [the] sws mutants. Conclusions. We found at least three genes-modulator of sws-phenotype, suggesting that reduced expression of these genes could play a neuroprotective role in toxicity- and stress induced neuropathies. At this time, the screening is going on. 


\title{
No association between RS1899663 hotair single nucleotide polymorphism and bladder cancer metastasis in ukrainian patients
}

\author{
Y. Chumachenko ${ }^{1}$, A. A. Roshchupkin ${ }^{1}$, A. V. Kolnoguz ${ }^{1}$, \\ A. D. Volkohon ${ }^{1}$, V. Yu. Harbuzova ${ }^{1}$ \\ ${ }^{1}$ Medical Institute of SSU
}

Background. To date long non-coding RNAs (lncRNAs) are actively studied since their regulatory potential in different biological processes was established, particularly, in transcription, translation, imprinting, cellular differentiation and development. The lncRNA HOTAIR (HOX antisense intergenic RNA) epigenetically affects the activity of cells cycle genes and its overexpression correlates with enhanced proliferation. Therefore, HOTAIR could be involved in [the] oncological transformation process, for instance, bladder cancer (BC) development. The aim of the research was to study the association between rs1899663 HOTAIR single nucleotide polymorphism (SNP) and BC metastasis in Ukrainian patients. Materials and methods. Venous blood of 141 patients with BC ( 68 patients with metastasis and 73 subjects without metastatic foci) was collected for genotyping. Polymerase chain reaction-restriction fragment length polymorphism (PCR-RFLP) was carried out in Thermocycler GeneAmp PCR System 2700 (Thermo Fisher Scientific, USA) for rs 1899663 HOTAIR allele's discrimination. The statistical processing of obtained results was done in the Statistical Package for Social Science software (SPSS, version 17.0, Chicago, IL, USA) and $\mathrm{P}<0.05$ was accepted as significant. Results. It was found the protective effect of TT-genotype against BC metastasis in both recessive $(\mathrm{Pc}=0.047$; $\mathrm{ORc}=0.334,95 \% \mathrm{CI}=0.113-0.986)$ and additive $(\mathrm{Pc}=0.04$; $\mathrm{ORc}=0.301,95 \% \mathrm{CI}=0.096-0.944)$ models of inheritance. However, after the adjustment for age, sex, smoking and alcohol consumption there was no significant association between rs1899663 HOTAIR genotypes and $\mathrm{BC}$ metastasis either in recessive $(\mathrm{Pa}=0.09 ; \mathrm{ORa}=0.386$, $95 \% \mathrm{CI}=0.129-1.159)$ or additive $(\mathrm{Pa}=0.076 ; \mathrm{ORa}=0.348,95 \% \mathrm{CI}=0.108-1.118)$ models. Conclusions. There is no association between rs 1899663 HOTAIR SNP and metastatic potential of BC in Ukrainian patients. 


\title{
Identification of genetic markers of the genetic variability of avian influenza a subtypes $h 1 \mathrm{n} 1$ and $h 7 n 9$. Determination of primers for the diagnostic method (lamp method)
}

\author{
S. Buriachenko ${ }^{1}$, B. T. Stegniy ${ }^{1}$ \\ ${ }^{1}$ NSC Institute of Experimental and Clinical Veterinary Medicine of the NAAS of Ukraine
}

Background. HA, NA, and NP genes are potential candidates as markers of genetic variability in avian influenza virus in birds, animals, and humans. Aim. The purpose of this study was to extract the potential of the HA, NA, and NP genes as markers of the polymorphism of avian influenza A viruses in domestic animals and humans with respect to the subtype H7N9 birds. Methods. The study used the MEGA6 and VectorNTI-11 Smith-Waterman algorithm nucleotide sequences of the hemaglutinin (HA), neurominidase (NA) and nucleoprotein (NP) genes taken from the virus database. A common region at the 3'-end of the segments was used to select the reverse primer sequences for amplifying the HA-NA and NP genes. To select direct primers, the multiple alignment of all the HA, NA, and NP gene sequences deposited in the ISD database (www.flu.lanl.gov) was performed. Then, the consensus sequences were formed for the HA and NA-ta NP genes; the common conservative areas (motifs) and selected sequences of direct primers were found for the first and second stages of amplification in compliance with the general principles; the primers were chosen. The following algorithm was used to select oligonucleotide markers capable of specifically detecting of a specific subtype of AIV: all HA, NA and NP sequences in the database were divided into groups according to certain variants of hemagglutinin ( $\mathrm{H} 1$ and $\mathrm{H} 7)$, neuraminidase ( $\mathrm{N} 1$ and $\mathrm{N} 9$ ) and nucleoprotein (NP). 40 resulting groups were divided into subgroups according to the origin of the virus (isolated from humans, birds, etc.), which formed a consensus sequence. Then, the most conservative motifs, limited by the primers of the second amplification stage, of the genomic segment, were determined. When it was impossible to identify a rather conservative motive, phylogenetic analysis of the sequences was performed within the subgroup with its division into smaller subgroups. Then a consensus sequence was formed and a search for conservative areas was conducted. Results. A total of 3500 sequences of hemagglutinin, neuraminidase, and AIV nucleoproein segments were analyzed. The variable structural regions of the nucleotide sequences of genes were determined. In all genes, single nucleotide substitutions are acquired, and the most polymorphic loci are located at the 3 'and 5' ends of the sequences. The number of oligonucleotides selected to determine a specific variant of HA, NA and NP depended on the number of identified conservative regions in the amplified region and on the degree of variability within the region. Based on the analysis, the primers were selected (4 for each gene of two subtypes) for an isothermal loop amplification reaction of viral genes, the set was constructed consisting of 24 discriminating oligonucleotides for specific analysis of AIV subtypes. Conclusions. The results of the analysis showed that the identification of RNA mutations and the most variable gene loci could be used for the genotyping of influenza A viruses. 


\title{
Smartphone-based sensor system for highly-selective detection of aflatoxin B1
}

\author{
D. Yarynka ${ }^{1}$, E. V. Piletska ${ }^{2}$, R. P. Linnik ${ }^{3}$, O. A. Zaporozhets 3 , O. O. Brovko ${ }^{4}$, \\ S. A. Piletsky², A. V. El'skaya1 ${ }^{1}$, T. A. Sergeyeva ${ }^{1}$ \\ ${ }^{1}$ Institute of Molecular Biology and Genetics, 150 Zabolotnogo str.,03143, Kyiv, Ukraine \\ ${ }^{2}$ University of Leicester, Chemistry Department, College of Science and Engineering, LE1 7RH Leicester, UK \\ ${ }^{3}$ Taras Shevchenko National University of Kyiv, Department of Chemistry, \\ 64/13 Volodymyrska Street, 01601, Kyiv, Ukraine \\ ${ }^{4}$ Institute of Macromolecular Chemistry, 48 Kharkivske Shosse, 02160, Kyiv, Ukraine
}

\begin{abstract}
Aim. Development of an easy-to-use smartphone-based fluorescent sensor system using molecularly imprinted polymer (MIP) membranes for rapid and accurate detection of widespread food mycotoxin - aflatoxin B1; optimisation of composition of the MIP membranes used as recognition elements of the sensor systems, and application of the sensor system for analysis of both model and real samples (food products and feeding stuff). Methods. The technique of molecular imprinting and method of in situ polymerization were used for molecularly imprinted polymer membranes synthesis. The MIP membranes were synthesized using nontoxic and non-fluorescent close structural analogue of aflatoxin B1 (ethyl-2-oxocyclopentanecarboxylate) as a dummy template. Triethyleneglycoldimethacrylate was used as a cross-linker. Compositions of MIP membranes were optimized using the method of computational modelling. Results. The quantitative detection of aflatoxin B1, selectively adsorbed by the MIP membranes, is based on its natural fluorescence. Sensor responses were registered after selective adsorption of mycotoxins by the nanostructured receptor sites in the MIP membranes' structure and generated by the UV-irradiation, which initiates natural fluorescence of the adsorbed mycotoxin. After $1 \mathrm{~min}$ excitation the images of the polymeric membranes samples were taken by Meizu U 10 smartphone camera and further were analysed using a commercially-available application for a smartphone Spotxel ${ }^{\circledR}$ Reader (Sicasys Software GmbH, Germany) for Android 6.0+. The smartphone application transforms the intensity of staining in arbitrary units and gives a possibility to get [real-time] dependencies of fluorescence intensity on toxin concentration in the sample within real time. Smartphone-based sensor systems using nanostructured MIP membranes were developed for detection of fungal mycotoxin aflatoxin B1 in food products and feeding stuff. The MIP membranes with the optimized composition demonstrated significantly higher selectivity towards aflatoxin B1 than to its analogues (aflatoxin G2, ochratoxin A). Aflatoxin B1 can be detected within the range 15$500 \mathrm{ng} / \mathrm{ml}$, detection limit $15 \mathrm{ng} / \mathrm{ml}$ corresponds to the maximum permissible concentrations in food and feedstuff. Storage stability of the proposed sensor systems was assessed during 1 year storage at room temperature. Conclusions. The smartphone-based sensor system provides highly-selective and sensitive detection of aflatoxin B1 in both model and real (maize and wheat flour) samples. The newly developed sensor system provides sensitive, precise, and inexpensive analytical method for aflatoxin B1 detection. In comparison with traditional methods of mycotoxins detection, the proposed smartphone-based sensor systems provide a convenient point-of-care approach in food safety testing and can be used in non-laboratory conditions. Acknowledgement. Financial support from National Academy of Sciences of Ukraine.
\end{abstract}




\title{
The effect of scaffold protein itsn1 on the functioning of rna-binding protein SAM68
}

\author{
S. Pankivskyi ${ }^{1}$, N. V. Senchenko, P. B. Busko ${ }^{1}$, L. Hamon ${ }^{2}$, D. Pastre ${ }^{2}$, A. V. Rynditch ${ }^{1}$ \\ ${ }^{1}$ Institute of Molecular Biology and Genetics NAS Ukraine, \\ Kyiv, Ukraine \\ ${ }^{2}$ Université d'Evry-Val-d'Essonne, \\ Evry, France
}

Background. Evolutionary conserved scaffold protein ITSN1 involved in clathrin-mediated endocytosis and signaling was recently shown to undergo nuclear-cytoplasmic shuttling. Although ITSN1 was found to interact with RNA-binding protein SAM68, the functional role of the interaction is unknown. Aim. The aim of the work was to analyze the structural basis of the ITSN1-SSAM68 interaction and to examine the effect of ITSN1 on SAM68 functioning. Methods. For GST pull-down assay, ITSN1 SH3 domains fused to GST were used to precipitate endogenous SAM68 or GFP-fused truncated forms of SAM68 from HEK lysates. Western blot analysis with anti-SAM68 and anti-GFP antibodies was used to detect the proteins. For sedimentation assay, purified recombinant proteins GST-ITSN1SH3 and SAM68-His were incubated in binding buffer and centrifuged at low speed. The protein content in pellet and supernatant fractions was estimated by SDS-PAGE and Coomassie staining. For immunofluorescent microscopy, HeLa cells transiently transfected with GFP-ITSN1 were fixed and stained with anti-SAM68 and Alexa Fluor 594-conjugated antibodies. For ITSN1 knockdown, HeLa cells were infected with lentiviral particles encoding ITSN1-specific shRNA. The expression of SAM68-regulated transcripts was evaluated using quantitative real-time PCR. Results. Based on the results of GST pull-down assay, it was found that SAM68 interacted with ubiquitously expressed and neuron-specific isoforms of ITSN1 SH3A domain. The class II proline motif (PxxPxR) that interacts with the SH3A domain of ITSN1 is present in the N-terminal low complexity region of SAM68. Using expression constructs encoding truncated forms of SAM68, it was found that ITSN1 SH3 domains precipitated N-terminal fragment of SAM68 confirming that ITSN1 interacts with PPLPHR motif (38-44 aa) of SAM68. Next, it was shown that ITSN1 SH3 domains facilitated solubilization of SAM68 aggregates in vitro whereas the accumulation of overexpressed ITSN1 in HeLa cells nuclei abolish the formation of SAM68 nuclear bodies specific for some transformed cell lines. Finally, it was found that ITSN1 knockdown altered SAM68-mediated splicing events in HeLa cells, particularly significantly increasing the expression of the pro-oncogenic isoform of splicing factor ASF/SF2. Conclusions. The current work demonstrated that ITSN1-SAM68 interaction is mediated by the SH3A domain of ITSN1 and the N-terminal low-complexity region of SAM68. ITSN1 induces the dissociation of SAM68 aggregates in vitro and in vivo whereas ITSN1 knockdown in HeLa cells affects splicing events controlled by SAM68 suggesting the presence of a novel nucleuscytoplasm signaling crosstalk. 


\title{
Effect of resveratrol treatment on meiotic maturation of oocytes, viability and dna integrity of follicular cells
}

\author{
V. Sribna ${ }^{1}$, M. Stupchuk ${ }^{1}$, O. Kaleynykova ${ }^{1}$ \\ ${ }^{1}$ O.O.Bogomoletz Instytute of Physiology \\ Kyiv, Ukraine
}

Background. Premature ovarian failure (POF), which is an ovarian function disorder affecting women under 40 years of age, is actively studied. In accordance with contemporary concepts about the development of POF, the leading role is given to the deletion of autoimmune pathology. Until now, it is unclear whether the development of the autoimmune process is a primary cause of this disease or it is the result of chronic pathology. Glomerulonephritis, in particular glomerulonephritis of immune etiology, represents a serious problem for reproductive health of women. The reproductive function may be affected by both, the glomerular disease itself and glucocorticoid and cytostatic therapy. Among the known antioxidants, Resveratrol (RES) has received numerous approvals when used in various disease patterns, including oocytes. According to recent studies , the effect of RES on reproductive function in women under conditions of experimental glomerulonephritis has not been studied yet, which makes this research relevant today. The aim was to estimate under conditions of experimental glomerulonephritis the effect of Resveratrol treatment on oocyte passage of meiotic maturation stages - metaphase I and metaphase II, on the viability and integrity of DNA of cells of the follicular environment of oocytes as well as pre- and post-implantational embryonic mortality in mice. Experiments (two series) have been conducted on CBA/lac mice (coloring wool agouti; genotype - +, H-2k): 64 females (10 weeks, 20-22 g) and 12 males (25 weeks, 25-27 g). Experimental glomerulonephritis in mice was achieved by immunization of white laboratory mice of the first generation with a kidney antigen suspension derived from a parent. Animal immunization was carried out at the rate of $10 \mathrm{mkL}$ of suspension per $10 \mathrm{~g}$ of body weight according to the following scheme: 3 times intra-abdominal 1 time per day; re-immunization was carried out after 3 weeks with a single intra-abdominal treatment of the same dose. Resveratrol (R5010, Sigma-Aldrich, USA) was intra-peritoneal treatment 4 times: 1 time per day for 1 hour before immunization of animals with suspension of kidney antigen; as well as in 3 weeks once with the same dose $(50 \mathrm{mg} / \mathrm{kg}, 0.3 \mathrm{~mL})$. In this work we have found that under conditions of experimental glomerulonephritis the treatment of Resveratrol results in an increase in the percentage of oocytes that successfully undergo meiotic maturation, a decrease in the percentage of apoptotic and necrotic cells of the follicular environment of oocytes and a decrease of the post-implantation mortality rate of embryos in mice. 


\title{
Application of liposomes in treatment of infections caused by Staphylococcus Aureus
}

\author{
A. Kosiv ${ }^{1}$, Yu. V. Karlash ${ }^{1}$ \\ ${ }^{1}$ National University of Food Technologies, Kyiv, Ukraine
}

Introduction. A large number of antibiotics are devoid of the ability of cell membrane and bacterial biofilm penetration, and intracellular short time retention, which results in inadequate intracellular distribution and low intracellular concentration [1]. Currently, the most promising method of treating infections of methicillin-resistant $S$. aureus is the usage of nanoparticles as antibiotic carriers [2]. Here are some examples that show improvement of the antibacterial effect against $S$. aureus infections when nanoparticle systems are used: gentamicin with liposomes and poly(lactic-co-glycolic acid); liposomal daptomycin, azithromycin and ceftazidime; enrofloxacin, vancomycin and tilmicosin with solid lipid nanoparticles [1]. The aim of the study. To create a targeted delivery system of chloramphenicol against Staphylococcus aureus infections. Materials and methods. Review of literature data, comparative analysis. Results. The studies used dimyristoylphosphatidylcholine (DMPC) and deoxycholic acid (DA) liposomes (with vesicle size of 132 and $239 \mathrm{~nm}$, respectively). The results show that an inhibition zone about 2-fold higher was achieved by DA liposomes as compared to the free control (1.4 $\mathrm{mm}$ and $0.6 \mathrm{~mm}$ respectively); the local antimicrobial concentration of the drug that penetrates the skin also increases. DA liposomes had minimal toxicity toward keratinocytes and neutrophils, which indicates good biocompatibility. Liposomal chloramphenicol has enhanced antimicrobial properties, due to the expansion of surface area, the interaction between the liposome and bacterial membrane, and prolonged drug release. These liposomes have shown both extracellular and intracellular activity against $S$. aureus [2]. Conclusions. Liposomal chloramphenicol may be useful in treatment of infectious follicular diseases caused by $S$. aure$u s$, and encapsulation of other types of antibiotics makes it possible to improve the effectiveness of treatment of other diseases. References. 1. Zhou K, Li C, Chen D, Pan Y, Tao Y, Qu W, Liu Z, Wang X, Xie S. A review on nanosystems as an effective approach against infections of Staphylococcus aureus. Int J Nanomedicine. 2018; 13:7333-7347. Published 2018 Nov 9. doi: 10.2147/IJN.S169935. 2. Hsu CY, Yang SC, Sung CT, Weng YH, Fang JY. Anti-MRSA malleable liposomes carrying chloramphenicol for ameliorating hair follicle targeting. Int J Nanomedicine. 2017; 12:8227-8238. Published 2017 Nov 10. doi: 10.2147/IJN.S147226. 


\title{
Identification of pseudomonas syringae, isolated from infected leaves of sugar beets
}

\author{
K. Kukol ${ }^{1}$ \\ ${ }^{1}$ Institute of Plant Physiology and Genetics NAS Ukraine, Kyiv, Ukraine
}

Background. Sugar beets are affected by many pathogens that cause disruption of normal physiological functions, morphological and biological structure of plants, and it leads to their growing oppression. Phytopathogenic bacteria are able to cause diseases for the majority of cultivated and wild plant species. They cause different types of lesions - tumor, necrosis, wilting, spot, while lowering crop yield and worsening the quality of the obtained harvest. Leaf spot and stripe diseases are widespread among bacterial diseases of the leaf apparatus of sugar beet. Aim. That is why the purpose of our study was the isolation of pathogenic bacteria from sugar beet leaves with the characteristics of destruction, determination of their morphological and cultural-biochemical properties and their identification. Methods. To isolate the bacteria from the leaves, the bits that cover part of the affected and healthy tissue were cut, then rubbed in a mortar with water and used to sow on the surface of the potato agar. The bacteria were grown at $28{ }^{\circ} \mathrm{C}$ for 5 days. Morphological and biochemical characteristics of allocated isolates were determined using classical methods. Identification of allocated bacterial isolates was done by comparing their properties with collection strains and properties described in the Guide to bacterial determination. Results. The bacteriological analysis resulted in isolation and identification of various morphological types of bacteria isolates. A clear reaction of hypersensitivity on tobacco leaves was initiated by 7 isolates. The results of artificial inoculation of sugar beet plants in the field conditions showed that isolates cause the necrotic dark spot formation on the leaves, which gradually increases in size. Additionally, the tissues inside the spots get dry and fall out. It has been established that by such criteria as Gram staining, motility, cell shape, presence of fluorescent pigment, absence of oxidase, aerobic use of glucose, absence of nitrate reduction and the formation of indole and hydrogen sulfide, the identified strains are identical to Pseudomonas syringae. Based on these features, we determined that the investigated pathogenic bacteria are identical to P. syringae, as they produce levan, do not cause maceration of plant tissues. Conclusions. Based on the morphological, cultural and biochemical [properties,] the isolated bacteria are identified as members of the P. syringae species, which initiate the infectious process once they are on the leaves of sugar beet, leading to appearance of dark necrotic spots. Its biological properties were determined and received results were compared with the data of other researchers who also had isolated and investigated this species. The symptoms of infections that we observed on the sugar beet are similar to the symptoms typical for the disease known as bacterial leaf spot of sugar beets. 


\title{
Exposure to an organophosphate tocp drosophila melanogaster flies with altered activity of sws and its micrornas genes
}

\author{
M. Tkachuk ${ }^{1}$, N. P. Matiytsiv 1 \\ ${ }^{1}$ Ivan Franko National University of Lviv
}

Background. Some neuropathies are developed after exposure to toxic substances. One of the examples is organophosphate-induced delayed neuropathy (OPIDN), neurodegenerative syndrome results from [the] impact of some organophosphates (OPs) found in agricultural pesticides. Human Neuropathy Target Esterase(NTE), encoded by PNPLA6 is the primary target of the toxic OP compounds. The exact mechanism of PNPLA6 function in OPIDN remains unknown. The swiss cheese (sws) is Drosophila orthologue of NTE. The flies treated with the OP compound tri-ortho-cresyl phosphate (ToCP) show behavioral deficits and neurodegeneration. The Drosophila sws mutants are also characterized by progressive degeneration of adult nervous system, glial hyperwrapping and neuronal apoptosis. We used this genetic model to study the role of sws and its miRNAs in developing toxicity-induced neurodegeneration. Methods. We studied effects of an OP ToCP on adult $D$. melanogaster wild type Oregon R, flies with overexpression of the whole sequence of sws(UAS-sws), overexpression of miRNAs (UAS-miR-966 and UASmiR-281), involved in SWS regulation and flies with functional knockout(UAS-sws-RNAi) of this gene in glia(repo-Gal4) and in places of its expression(sws-Gal4) using the Gal4-UAS system. All fly stocks were maintained and raised under standard conditions at $25^{\circ} \mathrm{C}$. The flies were collected after eclosion, kept on standard food for 5 days and then treated with $32 \mathrm{mg} / \mathrm{ml}$ ToCP solution for 24 hours. Flies were transferred to a standard food vial after treatment and the vials exchanged every 2 days. Dead flies were counted at $2 \mathrm{~d}, 7 \mathrm{~d}$ and $14 \mathrm{~d}$. Survival was analyzed using GraphPad Prism 6 (Graphpad Software Inc., La Jolla, CA, USA). Log-rank test was used to estimate statistically significant difference. Results. We revealed delayed toxic effect of $32 \mathrm{mg} / \mathrm{ml}$ ToCP on adult wild type $D$. melanogaster flies $(\mathrm{p}=0.001)$. The results show that the flies with overexpression of the whole sequence of sws are sensitive to organophosphate as well as with functional knockout in glia ( $p=0131$ and $p=0.0001$ respectively). Flies with overexpression of both miRNAs (miR-966 and miR-281) in glia are also sensitive to $\operatorname{ToCP}(\mathrm{p}=0.0325$ and $\mathrm{p}=00020)$ which shows the similarity to sws functional knockout. However, overexpression of miRNAs in places of sws expression leads to resistance to OP. Conclusions. Our data show perspective of Drosophila sws-depended neurodegenerative model for the ToCP influence study. 


\title{
Polymorphism of the 5S rDNA intergenic spacer in A. Mellifera carnica and $A$. M. Macedonica
}

\author{
N. Roshka ${ }^{1}$, A. V. Cherevatov ${ }^{1}$, R. A. Volkov ${ }^{1}$ \\ ${ }^{1}$ Yuriy Fedkovych Chernivtsi National University
}

Background. The genes encoding the ribosomal RNA (rDNA), in particular, 5S rDNA represent a convenient model for studying molecular evolution in eukaryotes. Taking into consideration that in insects this genomic region still remains insufficiently studied, the aim of our experiments was to analyze the molecular organization and polymorphism of $5 \mathrm{~S}$ rDNA spacer region in different subspecies of honeybee. Methods. The bees used in our study were obtained from breading farms of Austria and Greece. Total DNA was extracted from the body of bee using CTAB. Amplification of $5 \mathrm{~S}$ rDNA repeats was performed using polymerase chain reaction (PCR) with primers RV0803 and RV1406 that are complementary to the conservative region coding for $5 \mathrm{~S}$ rRNA. Purified PCR products were cloned into the $\mathrm{pGEM}^{\mathrm{TM}}-\mathrm{T}$ vector (Promega тM). The recombinant plasmids containing the $5 \mathrm{~S}$ rDNA inserts were sequenced and analyzed using the Lasergene software package. Results. It was found that the sequence of the 5S rDNA coding region was identical for all analyzed clones. The length of the amplified portion of the coding region without primers was $80 \mathrm{bp}$, and it was identical to the 5S rDNA coding regions of honey bees available in GenBank database. In contrast to the coding region, the 5S rDNA intergenic spacer (IGS) of the analyzed clones demonstrated polymorphism in both structure and length. The obtained clones were compared to the sequences from the genome of $\mathrm{A}$. mellifera, which is presented in GenBank. The analysis revealed several clusters of 5S rRNA genes, which are located on the chromosome 17. It was found that the IGS sequences of several A. $m$. carnica 5S rDNA clones, which were obtained in our experiments (e.g., SKL47\#2 pl2 and SKL47\#2 pl3) show a high similarity with the 5S rDNA clusters No 1 and No 4 detected in GenBank. At the same time, clone SKL47\#2 pl4 demonstrate[s] a high similarity with the IGS characteristic for clusters No 2 and No 3, suggesting the presence of several variants of intergenic spacers in the genome of A. m. carnica. We also compared the rDNA clones obtained for subspecies $A$. m. carnica and A. m. macedonica. It was found that the IGS sequences of $A . m$. macedonica significantly differ from each other. The sequences comparison showed that the level of similarity between the IGS of both subspecies and that of GenBank ranged from 90 to $93 \%$, except for the A. m. macedonica clone $1 \#$ pl5, which appeared to be identical to the IGS variants from GenBank cluster No 3. Conclusions. The analysis of the molecular organization of the IGS region showed that several variants of 5S rDNA are present in the genomes of honeybee subspecies, A. m. carnica and A. m. macedonica. 


\title{
Genetic risk factors of smoking status and quantity in ukrainian population
}

\author{
Y. Borysovych ${ }^{1,2}$, E. V. Murlanova ${ }^{1,3}$, O. O. Zahorodnia ${ }^{2}$, V. V. Moseiko ${ }^{1,3}$, \\ N. R. Darvyshov ${ }^{2}$, V. V. Bashynska ${ }^{1,3}$, A. K. Koliada ${ }^{1,3}$, O. M. Vaiserman ${ }^{3}$ \\ ${ }^{1}$ Genetic Laboratory, Ltd. «Diagen», \\ Kyiv, Ukraine \\ ${ }^{2}$ Institute of High Technologies, Taras Shevchenko National University of Kyiv, \\ Kyiv, Ukraine \\ ${ }^{3}$ D. F. Chebotarev State Institute of Gerontology, \\ Kyiv, Ukraine
}

Background. Tobacco consumption is currently responsible for the death of about six million people across the world each year. The active substance for cigarette dependence is nicotine, a naturally occurring drug found in all forms of tobacco. Nicotine is as highly addictive as heroin and cocaine. According to the Ministry of Health of Ukraine and the Global Adult Tobacco Survey (GATS) in 2017, $20.1 \%$ of the Ukrainian population were daily smokers. The probability of initial use of nicotine and progression toward a pathologic pattern of use are influenced by interaction of genotype, gender, age, drug availability, parenting style, socioeconomic status, etc. The relative importance of these factors varies across the lifespan and at different stages of addiction. Aim. To identify SNPs in the genes associated with cigarette smoking and the heaviness for nicotine addiction among Ukrainian population. Methods. 171 volunteers (mean age $32.6 \pm 9.6$ years) from Ukraine with no psychiatric diagnoses were enrolled in the study. All individuals gave the informed consent, collected buccal epithelium, filled in [the Cigarette Dependence Scale] CDS-5 questionnaire. We selected SNPs in the following genes: DNMT3B (rs910083), HTR2A (rs6313), CYP2A6 (rs4105144), COMT (rs4680), BDNF (rs6265), CHRNA5 (rs16969968), which had been implicated in nicotine dependence. Individuals with high CDS-5 score (present time or former smokers) were defined as cases and people with low values were defined as controls. CDS-5 score and its part describing heaviness of smoking based on the consumption of cigarettes per day (CPD) were used as continuous characteristics among all individuals. The carriage of individual alleles/genotypes and their combinations were compared in case/control samples using APSampler software, which is based on Bayesian MCMC method and provides validation by means of Fisher's exact test. We have built generalized linear regression models in $\mathrm{R}$ to analyze continuous phenotypes. Associations were considered significant when the p-values for them were less than 0.05 (for single associations) or less than 0.01 (for allelic combinations) Results. Genotype frequencies for all studied loci in the Ukrainian population are in Hardy-Weinberg equilibrium and correspond to frequencies in European populations. The allele HTR2AC carriage was associated with nicotine dependence $(\mathrm{p}=0.011$, OR $[95 \% \mathrm{CI}]=2.28[1.18-4.41])$. In the generalized linear models HTR $2 A C$ allele was associated with heaviness of nicotine addiction $(p=0.046$ for CPD and $p=0.028$ for CDS-5). Conclusion. We demonstrated the association of HTR2A rs6313 with smoking status and quantity. The obtained data indicate the relationship between serotonergic system and nicotine addiction. 


\title{
A multi-locus genetic analysis of alcohol dependence in ukrainian population
}

\author{
O. Zahorodnia ${ }^{1,2}$, E. V. Murlanova ${ }^{1,4}$, Y. H. Borysovych ${ }^{1,2}$, V. V. Moseiko ${ }^{1,4}$, \\ D. P. Osichanska ${ }^{3}$, V. V. Bashynska ${ }^{1,4}$, A. K. Koliada ${ }^{1,4}$, O.M. Vaiserman ${ }^{4}$ \\ ${ }^{1}$ Genetic Laboratory, Ltd. «Diagen», Kyiv, Ukraine \\ ${ }^{2}$ Institute of High Technologies, Taras Schevchenko National University of Kyiv \\ ${ }^{3}$ MedLux center of rehabilitation medicine, Kyiv, Ukraine \\ ${ }^{4}$ D. F. Chebotarev State Institute of Gerontology, Kyiv, Ukraine)
}

Background. Alcohol is a psychoactive substance that exerts a toxic and detrimental influence on brain and other body organs. It is a risk factor for many chronic diseases such as liver dysfunctions, cancers, ulcers and gastrointestinal problems. According to the statistics released by WHO and the Ministry of Health of Ukraine, a Ukrainian, over the age of 15, consumed in 2016 at average 8.6 liters of pure alcohol. It is generally acknowledged that genetics, environmental surroundings, cultural background, and other factors make the essential contribution to emergence of alcohol dependence. The combination of multiple genetic polymorphisms increases the risk for alcohol dependence. The objective of current study was to identify the associations of polymorphic variants of genes and their combinations with susceptibility to alcohol dependence in Ukrainian population. Methods. We recruited 300 volunteers resident in Ukraine (mean age 32.6 \pm 9.6 years). All individuals gave the informed consent to participate in the study and filled in the questionnaires including AUDIT-C and CAGE screening tests. We collected buccal epithelium of the volunteers, isolated and analyzed DNA for SNPs in the GABRA2 (rs279858), BDNF (rs6265), DRD2 (rs1800497), OPRM1 (rs1799971), $D A T 1$ (rs28363170) genes and $A D H 1 B-A D H 1 C$ (rs1789891) intergenic locus. [The] Individuals with F10 diagnosis ("Alcohol abuse ") were defined as cases (54 people) and those who acquired low AUDIT-C and CAGE values were defined as controls. Additionally, AUDIT-C was used to seek the association with amount of alcohol consumed. In case of binary phenotypes, we performed data analysis via APSampler algorithm using Monte Carlo Markov Chain method and Bayesian nonparametric statistics with the aim to find out the phenotypic association with alleles, genotypes and their combinations as well as to verify the statistical significance with Fisher's exact test. For assessment of continuous phenotypes, we constructed Multiple General Linear Regression models in R. The associations were considered significant when the p-values for them were less than 0.05 (for single associations) or less than 0.01 (for allelic ones). Results. The alleles and genotypes of the studied genes have frequencies similar to other European populations and are in Hardy-Weinberg equilibrium. The carriage of allele $\mathrm{A}$ at $A D H 1 B-A D H 1 C$ locus is associated with an increased risk of alcohol abuse ( $\mathrm{p}=0.03, \mathrm{OR}$ $[95 \% \mathrm{CI}]=2.15[1.04-4.45])$. Likewise, [a] combination of allele $\mathrm{A}$ of $A D H 1 B-A D H 1 C$ and allele A of GABRA2 gene ( $\mathrm{p}=0.002$, OR [95\% CI] $=3.41[1.56-7.44])$ is associated with alcohol dependence (at higher significance). No associations with the alcohol consumption level were identified, probably as a consequence of insufficient sample size. Conclusions. We have shown an association of SNPs in ADH1B-ADH1C and GABRA2 loci with alcohol abuse in Ukrainians. The findings demonstrate polygenicity and gene-gene interactions in the alcohol dependence development. The results should be validated in a larger sample. 


\title{
Application of psba-trnh igs for dna barcoding in genus Lathyrus
}

\author{
A. Nigda ${ }^{1}$, Y. O. Tynkevich ${ }^{1}$, R. A. Volkov ${ }^{1}$ \\ ${ }^{1}$ Yuriy Fedkovich Chernivtsi National University, \\ Department of Molecular Genetics and Biotechnology
}

Background. The psbA-trnH intergenic spacer (IGS) is one of the most commonly used in the molecular taxonomy and DNA-barcoding regions of the chloroplast genome. The advantages of this region are the possibility of direct sequencing of PCR products, a small size that facilitates PCR amplification and a moderately high nucleotide polymorphism, compared to other fragments of the cpDNA. However, the sequences of psbA-trnH for many plants are still absent in databases, which makes it difficult to identify them. This, in particular, concerns Lathyrus venetus (Mill.) Wohlf - the Red list species of the legume flora of Ukraine. The aim of current study was to amplify and sequence the IGS psbA-trnH of this species and to estimate the possibility of using it for DNA barcoding. Methods. PCR amplification was performed using the Maxima Hot Start PCR Master Mix (Thermo Scientific) polymerase. For the amplification, the primers complementary to the flanking IGS regions of psbA and trnH genes were used. The primers annealing temperature was $61^{\circ} \mathrm{C}$. The obtained PCR products were sequenced and the nucleotide sequence was analyzed using bioinformatics methods. Finding the matches in the GenBank database was conducted using the Blast program. Results. The sequence of IGS psbA-trnH for $L$. venetus has the length of $241 \mathrm{bp}$. The search in the Genbank database showed that the sequence of IGS of $L$. niger L. is most closely related to L. venetus among the deposited sequences. The calculated percentage of similarity between IGS of these species was $98.7 \%$. The vast majority of other Lathyrus species have a similar percentage of similarity to IGS of $L$. venetus in the range of 97.1 to $97.9 \%$. In comparison to all the aforementioned species IGS psbA-trnH $L$. venetus has two oligonucleotide insertions and one polynucleotide deletion at the 3'-end. Unexpectedly, the same deletion was found to be characteristic for the IGS of the genus Pisum L. Also, several point nucleotide substitutions have been identified. In general, it is important for DNA-barcoding that the nucleotide sequence of IGS psbA-trnH allows a clear distinction between $L$. venetus and other species of Lathyrus genus. Conclusions. At the next stage of our study, there will be conducted a sequencing of IGS psbA-trnH for closely related to L. venetus, but more abundant species L. vernus (L.) Bernh., which often forms morphologically similar hybrids with $L$. venetus. This complicates the control of the latter. Also, in order to better identify the hybrid forms, we plan to pick up the DNA-barcode of nuclear localization and use them together. 


\section{Investigation of quercetin molecule by quantum chemistry methods}

\section{Protsenko}

Department of Molecular Biotechnology and Bioinformatics, Institute of High Technologies, Taras Shevchenko National University of Kyiv, 2-h Akademika Hlushkova Avenue, 03022, Kyiv, Ukraine

Background. Quercetin is a biologically active compound. It is widely used in medicine and pharmaceutics, in particular, for the prevention of cardiovascular diseases and some types of cancer [1]. Quercetin occurs in fruits such as apples, grapes, dark cherries and blueberries, and vegetables: onion, garlic, carrot, as well as in green tea and olive oil. Quercetin is also known as a good antioxidant [2]. Aim. In this paper, the author introduced a broad assumption about the use of quercetin. In particular, we can indicate that the presence of multifunctional bioactive compounds may owe to the formation of different conformations and strong intramolecular hydrogen bonds. Methods. Initial geometries of the quercetin molecule were fully optimized without any structural restrictions at the DFT B3LYP/6-311++G(d, p) theory level using Gaussian [3]. Taking 2 orientations for 5 hydroxyl groups along with 2 possible mutual orientations of hetero and modified phenyl rings one easily obtains a priori estimation of possible conformation number of 64 . Results. The ab initio investigation has shown the quercetin molecule can adopt any of 12 mirror symmetrical planar conformations. Electron energies were calculated at the MP2/6-311++G(d,p) theory level. To get a new conformation it is enough to change the torsion angles that contain hydroxyl groups and torsion angle between hetero and modified phenyl rings. To reveal intramolecular H-bonds in all the conformers, AIM method was used. To investigate the ways of quercetin molecule conformations [, the] interconversion suitable transition states were located by QST3 method. Conclusions. Comparing the data obtained for different conformations of the molecule quercetin, in a free state, can testify certain results: the value of energy corresponding to intramolecular H-bonds have the same order and are in a close range. Existence of strong intramolecular H-bonds in the quercetin molecule, the average energies of which are $3.46-5.82 \mathrm{kcal} / \mathrm{mol}$, can, in particular, explain quercetin's biological multifunctionality.

[1] Abdur Rauf, Muhammad Imran, Imtiaz Ali Khan, Mujeeb - ur - Rehman, Syed Amir Gilani, Zaffar Mehmood, Mohammad S. Mubarak, Anticancer potential of quercetin: A comprehensive review // Phytotherapy Research. - 2018. - 32 (11) - 2109-2130. [2] Satyendra Singh Baghel, Nikhil Shrivastava, Rajendra Singh Baghel, Preeti Agrawal, Sarlesh Rajput, A review of quercetin: Antioxidant and anticancer properties // WORLD JOURNAL OF PHARMACY AND PHARMACEUTICAL SCIENCES. - 2012. - Volume 1, Issue 1 - 146160. [3] Проценко І.О., Говорун Д.М. Конформаційні властивості молекули кверцетину: квантово-хімічне дослідження // Доповіді НАН України. - 2014. - №3 - 153-157. 


\title{
Cell selection of wheat (Triticum Aestivum L.) For drought tolerance
}

\author{
S. Pykalo, T. V. Yurchenko, N. I. Prokopik \\ The V.M. Remeslo Myronivka Institute of Wheat, NAAS of Ukraine
}

Background. Wheat is the dominant crop in temperate countries being used for human food and livestock feed. Stress factors especially drought negatively affect the plant growth and development and cause a sharp decrease in the plant productivity. Using biotechnology and cell selection in particular is a promising direction in creating drought-tolerant wheat genotypes. Aim. To conduct cell selection of winter bread wheat for drought tolerance with application of selective system based on mannitol. MethodsWinter bread wheat genotype Erytrospermum 60068 was studied. Callus tissue was obtained from shoot apex explants of 3-day-old sterile seedlings. For callus induction[, the] MS medium supplemented with 2,4-D with a concentration of $2.0 \mathrm{mg} / \mathrm{l}$ was used. Mannitol at concentrations of $0.2,0.4$ and $0.6 \mathrm{M}$ was used as a selective agent. Calli were planted in petri dishes (40 in each) in 5 replicates. In 3 weeks the calli were tested for proportion of live. To select tolerant cell lines both direct and gradual cell selection was carried out. Direct selection was carried out according to the scheme: $0.6 \mathrm{M}$ mannitol (3 passages) $\rightarrow$ MS basic medium (1 passage) $\rightarrow 0.6 \mathrm{M}$ mannitol (2 passages) $\rightarrow$ regeneration and rooting ( 2 passages). Gradual selection was carried out according to the scheme: $0.2 \mathrm{M}$ mannitol (1 passage) $\rightarrow 0.4 \mathrm{M}$ mannitol (1 passage) $\rightarrow 0.6 \mathrm{M}$ mannitol (1 passage) $\rightarrow$ MS basic medium (1 passage) $\rightarrow 0.6 \mathrm{M}$ mannitol (2 passages) $\rightarrow$ regeneration and rooting ( 2 passages). To induce morphogenesis the calli were transferred to MS medium supplemented with $1 \mathrm{mg} / \mathrm{l} \mathrm{BAP}$ and $0.5 \mathrm{mg} / 1 \mathrm{IAA}$. In 3 weeks they were transferred to a modified medium for rooting. Rooted plants were transferred to in vivo conditions to produce seed generation. The obtained experimental data were processed by statistical analysis methods. Results. It was found that under the direct selection on medium with $0.6 \mathrm{M}$ mannitol, $9.5 \%$ of resistant calli survived to the end of the 6th passage. Under gradual selection to the end of the 6 th passage $11.0 \%$ of living calli were fixed on the medium with $0.6 \mathrm{M}$ mannitol. Thus, 2 callus lines that grew on selective media with $0.6 \mathrm{M}$ mannitol were obtained. The frequency of shoot regeneration from the tolerant cell lines was $6.5-9.5 \%$. The formation of plant regenerants from calli took place through both gemmorhizogenesis and somatic embryogenesis. In general, 15 drought stress-tolerant somaclonal variants obtained by cell selection are a valuable breeding material. Plant regenerants from the resistant lines were induced and their rearing, rooting and transfer to in vivo conditions were optimized. Conclusions. A gradual in vitro selection was more effective, because more resistant callus forms were identified as a result of selection and more plant regenerants were obtained. The developed method of in vitro selection of wheat for drought tolerance can be used in further research as the elements of biotechnological and breeding programs. 


\title{
Selective expression of the P60-S6 kinase 1 isoform induces an epithelial-mesenchymal transition in breast cancer cells MCF-7
}

\author{
I. Zaiets, V. R. Kosach, V. V. Filonenko \\ Institute of Molecular Biology and Genetics of NAS of Ukraine
}

Introduction. Ribosomal protein S6 kinase 1 (S6K1) exerts its functions within a cell via the usage of various isoforms, including p60, p70 and p85. Aberrant S6K1 signaling was shown to be implicated in the development of breast cancer. Involvement of S6K1 in breast cancer has been studied in terms of function of the p70- and p85-S6K1 isoforms, but not p60-S6K1. To expand the notion about a role of the p60-S6K1 isoform in the breast cancer development we have recently generated [the] MCF-7 cells with differential expression of S6K1 isoforms, including p60+/p70-/p85- MCF-7 and p60-/p70-/p85- MCF-7. The MCF-7 cells with selective expression of $\mathrm{p} 60-\mathrm{S} 6 \mathrm{~K} 1$ isoform revealed significantly elevated rates of cell migration relative to the wild-type MCF-7 and p60-/p70-/p85- MCF-7 cells. An increased ability to invade the surrounding tissues and metastasize is a feature of highly aggressive breast cancers that is accompanied by epithelial-mesenchymal transition (EMT). Aim. To reveal a link between differential expression of S6K1 isoforms and the induction of EMT in the breast cancer cell line MCF-7. Methods. Fluorescent microscopy, immunofluorescence analysis, western blot, RT-qPCR. Results. The morphological analysis of the generated cell lines revealed that the p60+/p70-/p85- MCF-7 cells have an elongated fibroblast-like shape and lose their ability to form discrete patches in culture compared to both the p60-/p70-/p85- MCF-7 and wild-type cells. The $\mathrm{p} 60+/ \mathrm{p} 70-/ \mathrm{p} 85-\mathrm{MCF}-7$ cell line was found to express vimentin, a mesenchymal cell marker, as indicated by western blot and immunofluorescence analysis. In addition to upregulated levels of vimentin, the qPCR analysis also revealed elevated levels of N-cadherin (mesenchymal marker) and down-regulated expression of E-cadherin (epithelial marker) in the cells with selective expression of p60-S6K1 demonstrating features of the mesenchymallike phenotype of these cells. Meanwhile, p60-/p70-/p85- MCF-7 and wild-type MCF-7 showed the reverse expression levels of the indicated EMT marker genes. The results of qPCR also revealed the EMT transcription program switching in p60+/p70-/p85- MCF-7 since these cells have up-regulated expression of Twist1 and ZEB2 transcription factors which are critical for the initiation of the EMT program. These data imply that the p60+/p70-/p85- MCF-7 cells underwent EMT, whereas p60-/p70-/p85 MCF-7 retained an epithelial-like phenotype. Conclusions. Differential expression of S6K1 isoforms under the conditions of p70- and/or p85-S6K1 knockdown and retaining of p60-S6K1 expression initiates EMT in the breast cancer cells MCF-7. This observation indicates a possible role of the p60-S6K1 isoform in the induction of EMT, and this function of p60-S6K1 is likely to be suppressed by the expression of $\mathrm{p} 70$ - and/or $\mathrm{p} 85-\mathrm{S} 6 \mathrm{~K} 1$ isoforms. 


\title{
Current state and prospects of bioinformatics development
}

\author{
O. Morozova, H. O. Chovpan, L. V. Batyuk \\ Kharkiv National Medical University
}

Background. Bioinformatics is usually understood as usage of computer technology for solution of biological problems, such as studying the specific algorithms and methods for analyzing large amounts of data, working mainly with genomic and protein sequences. Main reasons for the development of bioinformatics as a science were factors such as the development of medical biology and the necessity to create arrays of data obtained by experimental methods of medical biology. The aim of the work is to analyze the state of bioinformatics as a branch of science and to identify the prospects for its development. The methods are the exploration of the problems and achievements of bioinformatics and the definition of promising directions for the development of science in future. Results. The current development of molecular biology requires the active using of effective data analysis methods and usage of modern information technologies. In connection with this, there was a need to solve such practical tasks as the necessarily for work with a large amount of data, complex calculations and special requirements for the performance of computer technology. Bioinformatics uses methods of applied mathematics, statistics, theory and history, computer science and heuristic methods. Basics of the research areas of bioinformatics are alignment of sequences of genomes, gene search, a collection of genomes, alignment of protein structures, anticipating the structure of proteins, prediction of gene expression and protein-protein interaction and reconstruction of the evolution process. A special place has the receipt of high-quality sequences of genomes with fragments of sequences obtained using traditional DNA sequencing methods and designing of accounting for the strong factors for DNA-microchip data. An important role in bioinformatics plays the visualization of research results, such as browser of genomes. The genome is a one-dimensional map that displays any nucleotide awareness. Information is usually structured into blocks which are superimposed on tracks. The most popular are the Integrated Genome and the Browser Integrative Genomic Viewer. Results The development of bioinformatics has allowed us to quickly and reliably decode the human genome. Due to the achievements of bioinformatics that were able to create an artificial bacterium, it was possible to cure some types of cancer. Considering the specifics of data processing operations in bioinformatics and their volume, it seems effective the solution to the wide application of parallel computing technology to significantly improve productivity in solving many problems, in particular genome alignment, their search and assembly, preservation, and the ability to visualize the results of the researchers. 


\title{
DNA fragmentation of cryopreserved human sperm in men with patospermia
}

\author{
H. Hapon ${ }^{1}$, T. A. Yurchuk ${ }^{1}$, E. V. Pavlovich ${ }^{1}$, M. P. Petrushko \\ ${ }^{1}$ Institute for Problems of Cryobiology and Cryomedicine of the \\ National Academy of Sciences of Ukraine \\ ${ }^{2}$ Institute for Problems of Cryobiology and Cryomedicine of the National Academy of Sciences of Ukraine, \\ Medical Center "ART - Clinic of Reproductive Medicine"
}

Introduction. The level of sperm DNA integrity has an independent diagnostic and prognostic value for patients undergoing the infertility treatment using assisted reproductive technology (ART). According to WHO standards, the amount of sperm with damaged DNA should not exceed $30 \%$ in men with normozoospermia (WHO, 2010). An integral part of ART is spermatozoa cryopreservation which can lead to appearance of additional damages of DNA structure that cause not only a decrease of their fertilizing abilities, but also the abnormal development of the embryo derived from the in vitro fertilization. Aim. The aim was to study the influence of cryopreservation factors on the sperm DNA fragmentation level in the case of patospermia. Methods. The study included the data obtained during diagnostics of men, undergoing infertility treatment at the Medical Center "Clinic of Reproductive Medicine of ART" with their written informed consent. The research groups included ejaculatory spermatozoa obtained from men with oligoasthenoteratozoospermia (group 2) and testicular spermatozoa of men with obstructive azoospermia obtained by epididymis aspiration or extraction (group 3 ). The comparison group was spermatozoa of men with normozoospermia (group 1). Sperm cryopreservation was carried out by two-step method using $7 \%$ glycerol. The level of DNA fragmentation in sperm was determined using fluorescence microscopy and acridine orange staining. Results. It has been established that the number of spermatozoa with damaged DNA in ejaculatory spermatozoa was higher than in testicular ones. Thus, the level of DNA fragmentation in group 1 was $(17.5 \pm 3.02) \%$, in group $2(32.7 \pm 7.7) \%$ and in group 3 was $(6.2$ $\pm 1.3) \%$. After cryopreservation this indicator increased in all studied groups and amounted to $(27.2 \pm 1.9) \%,(41.6 \pm 5.7) \%$ and $(10.8 \pm 2.3) \%$ for groups $1-3$, respectively. Conclusions. The level of DNA fragmentation is higher in freshly isolated spermatozoa from oligoasthenoteratozoospermic men than in case of normozoospermia and obstructive azoospermia. Testicular sperm cells are characterized by a low level of DNA fragmentation. Cryopreservation causes an increase in the level of sperm DNA fragmentation regardless of the spermatogenesis state. The results of research should be confirmed during the infertility treatment by ART methods. 


\title{
Cultivation of newborn rabbit vibrissa dermal papilla cells in vitro
}

\author{
O. Novikova, G. A. Bozhok, T. P. Bondarenko \\ Institute for Problems of Cryobiology and Cryomedicine of the NAS of Ukraine
}

Background. The dermal papilla (DP) formation is associated with a complex process of neural crest cells migration. DP contains pluripotent cells that support renewal of a hair follicle over a long period. The possibility of culturing DP cells of several species (mouse, rat, human, and sheep) was shown. The aim of our study was to demonstrate the possibility of culturing DP cells of rabbit vibrissae. Methods. Cell cultures were isolated from explants by the method of Sieber-Blum, 2004. The isolated DP were placed in 6-well culture plates (PAA, Austria) and passaged every 7 days. It was investigated the possibility of obtaining different types of culture, such as monolayer and 3D culture of multicellular spheroids (MSs). The monolayer was cultured in the medium DMEM/F12 with $10 \%$ PBS (Biovest, France). This type of culture has a high proliferative potential; the proliferation rate on day 3 is 9-12. However, it is believed that the 3D cell culture of multicellular spheroids is the form in which cells better retain their uncommitted state (Santana et al., 2012). To obtain MSs, we used Petri dishes with low-adhesion surface (PAA, Austria). The DMEM/F12 culture medium with addition of $2 \%$ [of] supplements (B27 or Neuromax (Pan Eco, RF) was used in this case. Results. The formation of MS was observed when using both growth supplements. The formation of spheroids occurred by aggregation of single floating cells. The clusters formation started in several hours after passaging, a day later they contained 10-15 cells and acquired a spherical shape. During culture, the MSs were compacted and reached [the] sizes of 100-150 nm in 14 days. It was found that the cells in MS preserved the ability to grow in a monolayer. MS was transferred to [the] culture medium supplemented with $10 \%$ FBS on days 10-14 of cultivation, after which the adhesion cells started to migrate onto the substrate. Conclusions. Thus, the possibility of culturing DP cells of the vibrissa rabbit in vitro was shown. It is possible to obtain both monolayer and MS culture using different growth factors. The possibility to use Neuromax for this type of culture as an alternative to the frequently used nutritional supplement B27 was also shown. 


\title{
The usage of mesenchymal stem cells and bioceramics in bone tissue bioengineering
}

\author{
Y. Haluza ${ }^{1,2}$, V. V. Balatskyi ${ }^{1}$, O. O. Piven ${ }^{1}$ \\ ${ }^{1}$ Institute of Molecular Biology and Genetics of the National Academy of Sciences of Ukraine \\ ${ }^{2}$ ESC "Institute of Biology and Medicine", Taras Shevchenko National University of Kyiv, \\ Kyiv, Ukraine
}

Background. The number of bone disorders increases all around the world annually, so the development of effective methods for the bone tissue reconstruction is required. A combination of modern achievements in cell engineering and materials science provides an opportunity to create bone implants with the biological characteristics that would ensure complete regeneration of injured bone tissue. The new generation grafts use bioactive ceramics as a scaffold, which is an analogue of human bone tissue, and osteogenic cells to form a bone matrix. Mesenchymal stem cells (MSCs) are perspective candidates for those kind cell-based therapies because of their regenerative characteristics and ability to differentiate into osteoblasts. We have focused on adipose tissue-derived MSCs (ASCs) as they are easily accessible source of autologous osteogenic cells. Our main goal is to create promising methodological approaches for new generation of bone tissue graft creation. For this we have addressed to optimization of isolation of MSCs from adipose tissue, co-cultivation them with bioactive ceramic and analysis of osteogenic differentiation under experiment conditions. Methods. We have used methods of cell biology and molecular genetics. MSCs isolation from rats adipose tissue. ASCs cultivation in osteogenic medium with bioactive ceramics granules: Biomin GTG-1 (consists of $\beta$-tricalcium phosphate and hydroxyapatite), Biomin T-500 (consists of $\beta$-tricalcium phosphate) at $37^{\circ} \mathrm{C}, 5 \% \mathrm{CO} 2$. Metabolic activity of MSCs was determined by MTT test. Flow Cytometry (FACS) Analysis of expression of positive markers CD105, CD73, CD90 and negative CD34, CD 45, CD31, CD145, CD114 that are known to be expressed by ASCs. Quantitation of osteopontin, osteocalcin, main osteogenic markers, mRNA levels by real-time PCR. **Results. ${ }^{* *}$ In result of work we have optimized the methods ASCs isolation, FACS analysis confirm the presence of a common set of CD markers expressed in stromal cells from adipose tissue e.g. CD90, CD73 (>95\%), CD105 (<2\%). MSCs are negative for hemaptopoietic cell markers: $\mathrm{CD} 45, \mathrm{CD} 34(<0.2 \%)$. Next, we have analyzed the biocapacity and influence of bioactive ceramics on cells proliferation: Biomin GTG-1, Biomin T-500. In result we have found that only GTG-1 and T-500 don't limit cells growth. After 3 weeks of osteogenic differentiation with bioactive ceramics, qRT-PCR revealed an upregulation of osteogenic markers osteopontin, osteocalcin for ASCs. Conclusions. So, ASCs demonstrate a high potential for their use in bone grafts engineering. Our results showed that bioactive ceramics enhance ASCs osteogenic differentiation, so "T-500", "GTG-1" can be used as a new biocompatible scaffold for bone regeneration. 


\title{
HSA-MIR-30A-5P and HSA-MIR-200C-3P expression in tumor and patient blood plasma as diagnostic biomarker of clear cell renal cell carcinoma
}

\author{
K. Onyshchenko ${ }^{1}$, T. V. Voitsitskyi ${ }^{1}$, Grygorenko V. M. ${ }^{2}$, L. V. Pereta ${ }^{2}$, I. Ya. Skrypkina ${ }^{1}$ \\ ${ }^{1}$ Institute of Molecular Biology and Genetics of the National Academy of Sciences of Ukraine, \\ Kyiv, Ukraine \\ ${ }^{2}$ Institute of Urology, National Academy of Medical Sciences of Ukraine, \\ Kyiv, Ukraine
}

Background. Clear cell renal cell carcinoma (ccRCC) is the most common subtype detected in $70-80 \%$ of patients with renal cell carcinoma (Lopez-Beltran, 2006). However, due to complicated diagnostics, in 40-50\% of patients ccRCC is found after the metastases generation (Shinmei, 2013). Hence, there is an urgent need to develop novel, more effective methods for the ccRCC diagnostics. Many studies have shown that miRNAs take part in several processes of tumorigenesis, involving proliferation, migration, invasion, and metastasis (Fendler, 2011), which suggests potential values of miRNAs as the disease biomarkers. As biomarker candidates two miRNAs were chosen: miR-200c-3p which is known to play crucial roles in epithelialmesenchymal transition, cell invasion, proliferation, metastasis, and therapy resistance in several cancer types (Humphries, 2015, Mutlu, 2015), and miR-30a-5p, the cancerogenic role of which has been explored in various cancer types including ccRCC (Sun, 2019). Aim. Here we investigated the possibility to use the miR-200c-3p and miR-30a-5p expression level in blood plasma samples as a diagnostic biomarker. Methods. Tumor surgical specimens and control samples (non-malignant tissues) of kidney and blood plasma (taken before the surgery) were obtained from the Institute of Urology, NAMS of Ukraine. Total RNA from the tissues samples was isolated with TRI Reagent (Sigma, USA) and reverse transcribed by the HighSpecificity miRNA 1st-Strand cDNA Synthesis Kit (Agilent Technologies, USA) for quantitative real-time PCR determination of the miR-200c-3p and miR-30a-5p expression. Results. By comparison of tumor to control samples we found 5-fold downregulation of miR-30a-5p and 5.8-fold of miR-200c-3p with $\mathrm{AUC}=0.879$ and $\mathrm{AUC}=0.865$ respectively. Interestingly, [the] miR-200c-3p expression level in blood plasma was 7.9-fold lower than in tumor and 8.7-fold lower than in plasma of healthy donors. [The] miR-30a-5p expression level in blood plasma was 9.8-fold lower than in RCC tissues and 6.4- fold lower than the plasma levels of healthy donors. AUC of miR-30a-5p and miR-200c-3p in the blood plasma of the patients compared to controls was 0.969 and 0.973 indicating a highly significant diagnostic potential of this microRNA. Conclusions. Based on these results, we consider miR-30a-5p and miR$200 c-3 p$ as a promising marker of ccRCC early diagnostics.

Acknowledgment. The research was supported by the grant of the National Academy of Sciences of Ukraine, competition project 115U002951. 


\title{
Physiological and biochemical properties of chickpea nodule bacteria with different growth rates
}

\author{
O. Lohosha, Y. O. Vorobey, T. O. Usmanova \\ Institute of Agricultural Microbiology and Agroindustrial Manufacture NAAS
}

\begin{abstract}
Aim. Study of morphological, cultural, physiological and biochemical properties of strains of chickpea nodule bacteria. Methods. Isolated new strains of rhizobia from pink nodules roots of chickpea. Plants were selected in the flowering phase. Cultural, morphological, physiological and biochemical properties were determined by standard microbiological methods, as well as using light and electron microscopy. Results. 58 bacterial isolates were isolated from nodules of chickpea varieties Skarb, Admiral, Odysei, Budzhak, Triumf and Pam'iat' grown in the steppe zone of Ukraine (Odessa region). In the soil of these fields, an active rhizobia population has been formed due to the long-term cultivation of this crop. Other 11 isolates from the plant nodules of varieties Triumf and Pam'iat' selected in the forest-steppe zone (Lviv region), in the fields where chickpeas were grown for the first time. The ability of the obtained isolates to form nodules was studied under the conditions of vegetation experiments in sterile vermiculite. It was shown that all the studied strains capable of chickpea nodulation correspond to the morphological characteristics of the genus Mesorhizobium: gram-negative aerobic movable rods do not form spores, cells 1.0-2.0 x 0.3-0.5 $\mu \mathrm{m}$ in size. However, the cultural properties of the studied strains differed significantly. So, of all the strains of rhizobia isolated from chickpea nodules of different varieties, in different soil and climatic conditions, some belong to the "fast-growers". On bean agar, these strains form sufficiently large colonies with a diameter of 2-4 mm for 2-3 days. Noteworthy, a significant part of isolates obtained from areas of long-term chickpea cultivation were "meso-growers" strains that form small colonies (1-2 mm) on bean agar for 4-7 days of cultivation. Physiological and biochemical studies of Mesorhizobium sp. strains showed that "fast-growers" strains do not use D-arabinose and sorbitol as a source of carbohydrates, and when grown with mannitol and dulcite they acidify the medium. "Mesogrowers" strains do not change the reaction of the medium with dulcite, moderately acidify the medium with mannitol and sorbitol. It was established that the studied fast-growing strains are characterized by abundant growth on media with ammonium phosphate and on meatpeptone agar. The strains with an average growth rate use ammonium molybdate as a source of nitrogen, and are also characterized by poor growth by MPA or its absence. Conclusions. It was shown that the strains of Mesorhizobium sp. isolated from chickpea nodules grown in different soil-climatic zones of Ukraine, have similar cell morphology, but differ in growth rate, colony size, physiological and biochemical properties.
\end{abstract}




\title{
The effect of MN2+ and LA3+ ions on functioning of lcc-channels in the nuclear membrane
}

\author{
I. Yuryshynets ${ }^{1,2}$, A. B. Kotliarova ${ }^{1}$, O. A. Kotyk ${ }^{1}$, S. M. Marchenko ${ }^{1}$ \\ ${ }^{1}$ Bogomoletz Institute of Physiology National Academy of Science of Ukraine \\ ${ }^{2}$ ESC "Institute of Biology and Medicine", Taras Shevchenko National University of Kyiv
}

Background. The contemporary research data confirm that ion channels with distinct biophysical properties are localized in the nuclear envelope, but physiological role and structure of LCC-channels of nuclear membrane are not fully elucidated. The aim of this work was to investigate the influence of metal ions on the LCC-channels functioning in the nuclear membrane of Purkinje neurons. Methods. The experiments were carried out on 21-days old Wistar rats. The isolation of the Purkinje neurons nuclei was carried out by the method developed by Marchenko S.M. Ion currents through the channel were recorded using patch clamp technique. The results have been analyzed using the Clampfit and Origin software. Results. According to the preliminary data obtained in our laboratory large monovalent cations $(\mathrm{K}+, \mathrm{Na}+)$ penetrate the LCC-channels whereas divalent $(\mathrm{Ca} 2+)$ and trivalent $(\mathrm{La} 3+)$ - do not. It is known that ions of metals pass through the channels in a hydrated state. For the divalent ions, hydrated envelope is larger than in monovalent ones. In turn, trivalent cations bind more $\mathrm{H} 2 \mathrm{O}$ molecules, and as a result, the effective size of their complexes with water is larger than that of divalent. We have investigated the effect of ions $\mathrm{Mn} 2+$ and $\mathrm{La} 3+$ at concentrations of 1-10 $\mathrm{mM}$. It was found that $10 \mathrm{mM} \mathrm{Mn} 2+$ moderately (by 1-2 pA) reduced the average current through the channel without affecting their open probability. La3+ ions at the same concentration inhibited LCCchannel functioning more effective, reducing the average current through the channel by 4-5 pA and increasing the frequency of its being in the closed state (channel flickering). In both cases, the effects were reversed. We also revealed that the more negative the value of the applied potential, the more effectively the ion reduces the current through the LCC-channel. At the same time, for the potential $-60 \mathrm{mV}$, the effect of $10 \mathrm{mM} \mathrm{Mn} 2+$ solution on average increased twice in comparison to the effect at $-40 \mathrm{mV}$. At a value $-60 \mathrm{mV}$, the current through the LCC-channel under the influence of $\mathrm{La} 3+$ was reduced by $75 \%$, and at $-20 \mathrm{mV}$ its effect was $56 \%$. At positive potential values $(+20-+80 \mathrm{mV})$, the average effect of both $\mathrm{Mn} 2+$ and La3+ was insignificant. Additionally, we conducted experiments on the influence of Pt metal in the complex compound - cisplatin. It has been revealed that in this form, this substance did not affect the functioning of LCC-channels. Conclusions. Thus, the electrophoretic mobility of metal ions can be used as indicator of their real size (with a hydrated envelope) in aqueous solutions. Application of metal ions as a research tool makes it possible to estimate the pore diameter of the ion channel and determine the possible binding sites using the methodological approaches of molecular dynamics. Acknowledgment. Research group would like to express their gratitude to Mr. Shota Khajishvili for sponsoring this project. 


\title{
Potential-dependence of the functioning of the IP3RS in the native nuclear envelope of cardiomyocytes
}

\author{
N. Pavlova ${ }^{1,2}$, O. A. Kotyk ${ }^{1}$, A. B. Kotliarova ${ }^{1}$, S. M. Marchenko ${ }^{1}$ \\ ${ }^{1}$ Bogomoletz Institute of Physiology National Academy of Science of Ukraine \\ ${ }^{2}$ ESC “Institute of Biology and Medicine”, Taras Shevchenko National University of Kyiv
}

Background. The inositol 1,4,5-trisphosphate receptors (IP3Rs) are involved in intracellular $\mathrm{Ca} 2+$ signaling and provide output of calcium ions from a $\mathrm{Ca} 2+$-store in response the IP3 concentration increase. These receptors are widely described in the endoplasmic reticulum but only few data about their properties in a nuclear membrane are known. The aim of the work was to estimate the potential-dependence of the IP3R functioning in the native nuclear membrane of cardiomyocytes. Methods. In the experiments 2-3 week-old Wistar and Fisher rats were used. The nuclei of cardiomyocytes were isolated by myocardium homogenization and centrifugation of the resulting suspension. Single ion channels were recorded from nucleusattached and excised patches of the nuclear membrane in the voltage-clamp mode of the patch-clamp technique. The bath and glass pipettes were filled with solution containing $(\mathrm{mM})$ : KCl-150, HEPES-8, HEPES-K-12, EGTA-1, IP3 0.002, ATP 0.5 (pH 7.2). Results. In the native nuclear membrane of cardiomyocytes we have registered inositol-1,4,5-triphosphate receptors with conductance $384 \pm 5 \mathrm{pS}$. They were characterized by a significant dependence on the membrane potential, the open probability of IP3 receptors at positive potentials is much higher than at negative. The dependence of IP3 receptors on the potential may be the base of the mechanisms for regulating the duration of calcium signals in the nucleus. $\mathrm{Ca} 2+$ release from the stores leads to local membrane potential changes. Long-term $\mathrm{Ca} 2+$ release requires compensation of these changes with different additional mechanisms. Previously, we described LCC-channels in cardiomyocytes nuclear membrane. They are involved in maintanace of the membrane potential, creating a flow of $\mathrm{K}+$ ions through a nuclear membrane. Due to the fact that IP3Rs and LCC-channels are coexpressed in the cardiomyocytes nuclear membrane, we believe that LCC-channels may stabilize the local nuclear membrane potential and facilitate the $\mathrm{Ca} 2+$ release. Conclusions. Consequently, we found that inositol-1,4,5-triphosphate receptors functioning in the native nuclear membrane of cardiomyocytes depend on the membrane potential, at positive potentials they showed the highest activity, whereas at negative potentials their activity decreased. This may be important for regulating the duration of calcium signals in the nucleus. Acknowledgments. The publication contains the results of studies supported by the President's of Ukraine grant for competitive projects (project $\Phi 75 / 29460$ ) of the State Fund for Fundamental Research. Borosilicate glass was partially sponsored by Mr. Shota Khajishvili. We wish to acknowledge the help provided by A. Polishchuk at the initial stages of the project. 


\title{
Fluorescence and circular dichroism spectroscopy studies of an interaction between interferon $\alpha 2 \beta$, oligoribonucleotides and amp
}

\author{
R. Nikolaiev, S. I. Chernykh, Z. Yu. Tkachuk \\ Institute of Molecular Biology and Genetics of NAS of Ukraine
}

Background. Interactions of protein-nucleic acids play a decisive role in many biological processes. Aim. We studied the ability of yeast oligoribonucleotides RNA (ORNs), yeast oligoribonucleotides RNANa salt (ORNsNa), yeast oligoribonucleotides-D-mannitol complex (ORNs-D-M) and RNANa with mannitol (ORNsNa-D-M) to affect thefluorescence quenching and conformational changes of Interferon $\alpha 2 \mathrm{~b}$. We also conducted the research with AMP. Methods. To investigate the interaction and conformational changes of IFN protein, the fluorescence and CD spectroscopies were used. The IFN secondary structure changes were analyzed by service Bestsel. Results. The dissociation constant $\mathrm{Kd}=1.11 \pm 0.1 \mu \mathrm{M}$ was observed in the fluorescence quenching interaction between INF and ORNs-D-M. The dissociation constant between IFN and ORNs is $\mathrm{Kd}=2.36 \pm 0.5 \mu \mathrm{M}$, between INF and ORNsNa $-\mathrm{Kd}=2.15 \pm 0.16 \mu \mathrm{M}$ and INF and ORNsNa-D-M Kd=3.13 $\pm 0.5 \mu \mathrm{M}$. The dissociation constant $\mathrm{Kd}=1.74 \pm 0.09 \mu \mathrm{M}$ was observed in interaction between INF and AMP-D-M. The dissociation constant between IFN and AMP is $\mathrm{Kd}=7.95 \pm 0.4 \mu \mathrm{M}$, between INF and AMPNa $-\mathrm{Kd}=12.1 \pm 0.2 \mu \mathrm{M}$ and INF and AMPNa-D-M Kd=9.4 $\pm 0.7 \mu \mathrm{M}$. The analysis of IFN secondary structure shows that regular $\alpha$-helix components (r $\alpha$-h) $16.7 \%$, distored $\alpha$-helix components $(\mathrm{d} \alpha$-h) $10.3 \%$, antiparallelleft $\beta$-stand (al $\beta$-s) $2.9 \%$, antiparallelrelaxed $\beta$-stand $(\operatorname{arx} \beta$-s) $16 \%$, antiparallelright $\beta$-stand $(\operatorname{ar} \beta$-s) $10.2 \%$, parallel $\beta$-stand $(\mathrm{p} \beta$-s) $0 \%, \beta$-turn $8.1 \%$ and random coil components (r.c.c.) $35.8 \%$. Adding of ORNs shows that r $\alpha-\mathrm{h} 26.9 \%$, d $\alpha$-h $12.8 \%$, al $\beta$-s $0 \%$, arx $\beta$-s $11.9 \%$, $\operatorname{ar} \beta$-s $0 \%$, p $\beta$-s $0 \%$, $\beta$-turn $8.2 \%$ r.c.c. $40.2 \%$, to wit increase $\alpha$-helix components in comparison with native protein. Adding of ORNs-D-M shows that r $\alpha$-h $23 \%$, d $\alpha$-h $10.4 \%$, al $\beta$-s $0.4 \%$, $\operatorname{arx} \beta$-s $14.5 \%$, $\operatorname{ar} \beta$-s $0 \%$, p $\beta$-s $0 \%$, $\beta$-turn $8.2 \%$ r.c.c. $43.3 \%$, to wit decrease a $\beta$-s in comparison with native INF. On the other hand, adding ORNsNa leads to r $\alpha$-h $0.7 \%$, d $\alpha$-h $10.8 \%$, al $\beta$-s $4.8 \%$, arx $\beta$-s $14.9 \%$, ar $\beta$-s $0 \%$, p $\beta$-s $0 \%$, $\beta$-turn $11.9 \%$ r.c.c. $36.9 \%$, to wit increase $\beta$-turn in comparison with native protein. Addition of ORNsNa-D-M leads to r $\alpha-\mathrm{h} 35.3 \%$, d $\alpha$-h $20.2 \%$, al $\beta$-s $0 \%$, arx $\beta$-s $0 \%$, ar $\beta$-s $5.2 \%$, p $\beta$-s $0 \%$, $\beta$-turn $2.8 \%$ r.c.c. $36.4 \%$, to wit increase $\alpha$-helix components. Addition of AMP shows that $\mathrm{r} \alpha-\mathrm{h} 0 \%$, d $\alpha$-h $1.4 \%$, al $\beta$-s $7.8 \%$, $\operatorname{arx} \beta$-s $6.2 \%$, ar $\beta$-s $32.4 \%$, p $\beta$-s $26 \%$, $\beta$-turn $0 \%$ r.c.c. $26.1 \%$. Addition of AMP-D-M shows that $\mathrm{r} \alpha$-h $0 \%$, d $\alpha$-h $0 \%$, al $\beta$-s $11.8 \%$, arx $\beta$-s $3.3 \%$, ar $\beta$-s $0 \%$, p $\beta$-s $23.2 \%$, $\beta$-turn $6 \%$ r.c.c. $55.7 \%$. On the other hand, adding AMPNa leads to r $\alpha$-h $0 \%$, d $\alpha$-h $0 \%$, al $\beta$-s $17.5 \%$, arx $\beta$-s $0 \%$, $\operatorname{ar} \beta$-s $16.5 \%$, p $\beta$-s $31.2 \%$, $\beta$-turn $1.1 \%$ r.c.c. $33.7 \%$. Addition of AMPNa-D-M leads to $\mathrm{r} \alpha$-h $29.9 \%$, d $\alpha$-h $5.8 \%$, al $\beta$-s $0 \%$, arx $\beta$-s $0 \%$, ar $\beta$-s $1.4 \%$, p $\beta$-s $11.3 \%$, $\beta$-turn $1.9 \%$ r.c.c. $48.8 \%$, to wit increase $\mathrm{p} \beta$-s. Conclusions. The ORNs and AMP have the advantage of interacting with proteins, unlike salts ORNs and AMP, because of a stronger binding. Different effects of different forms of ORNs and AMP on the secondary structure of the INF can be explained by various binding sites. 


\title{
The effect of tyrosinase inhibitor on hydrogen peroxide content in wheat leaves
}

\author{
I. Zhuk ${ }^{1}$, L. O. Kucherova ${ }^{2}$ \\ ${ }^{1}$ Institute of Plant Protection of NAAS of Ukraine \\ ${ }^{2}$ Institute of Cell Biology and Genetic Engineering, Natl. Acad. Sci. Ukraine, \\ Kyiv, Ukraine/Institute of Plant Protection of Natl. Acad. Agrar. Sci. Ukraine
}

Background. Melanin, the product of tyrosinase activity, plays an important role in fungal pathogen Septoria tritici. Kojic acid as a tyrosinase inhibitor has a potential for elicitation of defense responses in plants. Biotic elicitors may induce the plant disease resistance and prevent increased environmental pollution by pesticides. Systemic acquired resistance to pathogens in plants is supposed to depend on $\mathrm{H} 2 \mathrm{O} 2$. In our previous research it has been shown that biotic elicitors influence the $\mathrm{H} 2 \mathrm{O} 2$ content in plants. The aim of research was to analyze in field the trials effect of kojic acid with an additional donor NO treatment on the hydrogen peroxide (HP) content in winter wheat leaves. Methods. Content of endogenous $\mathrm{H} 2 \mathrm{O} 2$ was measured in kojic acid and NO donor treated wheat plants (cv. Legenda Myronivska) at different ontogenesis phases. The extent of morphometric parameters and yield structure were analyzed.

Results. The data obtained suggest that kojic acid and donor NO decreased the HP content in wheat leaves and increased the grain number and yield. Hydrogen peroxide is a signal molecule for photosynthetic status and for stomatal movements. So kojic acid influences indirectly the assimilation processes. Conclusions. Kojic acid with donor NO could be used as a more effective combination than biotic elicitor.

Keyword s: winter wheat, kojic acid, NO, biotic elicitors, induced resistance, Triticum aestivum L., Septoria tritici 


\title{
Novel conformationally-tautomeric properties of the biologically important a.t dna base pairs
}

\author{
O. Brovarets', K. S. Tsiupa, D. M. Hovorun \\ Institute of Molecular Biology and Genetics of NAS of Ukraine
}

\begin{abstract}
Aim. To discover novel mechanisms of the conformational and tautomeric transitions of the classical A·T DNA base pairs - Watson-Crick A·T(WC), reverse Watson-Crick A·T(rWC), Hoogsteen $\mathrm{A} \cdot \mathrm{T}(\mathrm{H})$ and reverse Hoogsteen $\mathrm{A} \cdot \mathrm{T}(\mathrm{rH})$ - and their wobble conformers. Methods. MP2/aug-cc-pVDZ//B3LYP/6-311++G(d,p) level of QM theory. Results. For the first time, we have established that: - The $A \cdot T(W C) \leftrightarrow A \bullet T(w W C), A \cdot T(r W C) \leftrightarrow A \cdot T(w r W C)$, $\mathrm{A} \cdot \mathrm{T}(\mathrm{H}) \leftrightarrow \mathrm{A} \bullet \mathrm{T}(\mathrm{wH})$ and $\mathrm{A} \cdot \mathrm{T}(\mathrm{rH}) \leftrightarrow \mathrm{A} \bullet \mathrm{T}(\mathrm{wrH})$ conformational transformations (Gibbs free energies of activation $\Delta \mathrm{G}=7.13,7.26,7.67$ and $7.44 \mathrm{kcal} \cdot \mathrm{mol}-1$ in the continuum with $\varepsilon=4$ ) [1]. - The $\mathrm{A} \cdot \mathrm{T}(\mathrm{WC}) \leftrightarrow \mathrm{A} \cdot \mathrm{T}(\mathrm{H})$ and $\mathrm{A} \cdot \mathrm{T}(\mathrm{rWC}) \leftrightarrow \mathrm{A} \cdot \mathrm{T}(\mathrm{rH})$ structural transitions $(\Delta \mathrm{G}=1.97$ / $9.53 / 9.52$ and $1.84 / 9.12 / 9.28 \mathrm{kcal} \cdot \mathrm{mol}-1$ in the continuum with $\varepsilon=4)$ act as intermediates of the pairwise $\mathrm{A} \cdot \mathrm{T}(\mathrm{WC}) / \mathrm{A} \cdot \mathrm{T}(\mathrm{rWC}) \leftrightarrow \mathrm{A} \cdot \mathrm{T}(\mathrm{H}) / \mathrm{A} \cdot \mathrm{T}(\mathrm{rH})$ conformational transformations [2]. - The $A \cdot T(w H) \leftrightarrow A \cdot T(w r W C), A \cdot T(w W C) \leftrightarrow A \cdot T(w r H), A \cdot T(w W C) \leftrightarrow A \cdot T(w r W C)$ and $\mathrm{A} \cdot \mathrm{T}(\mathrm{wH}) \leftrightarrow \mathrm{A} \cdot \mathrm{T}(\mathrm{wrH})$ conformational transitions $(\Delta \mathrm{G}=3.20,3.70,12.04$ and $10.69 \mathrm{kcal} \cdot \mathrm{mol}-1$ in the continuum with $\varepsilon=1$ at $\mathrm{T}=298.15 \mathrm{~K}$ ) define the interconversions between the classical A.T DNA base pairs: $\mathrm{A} \cdot \mathrm{T}(\mathrm{WC}) \leftrightarrow \mathrm{A} \cdot \mathrm{T}(\mathrm{rWC}) / \mathrm{A} \cdot \mathrm{T}(\mathrm{rH})$ and $\mathrm{A} \cdot \mathrm{T}(\mathrm{H}) \leftrightarrow \mathrm{A} \cdot \mathrm{T}(\mathrm{rH}) / \mathrm{A} \cdot \mathrm{T}(\mathrm{rWC})$ [3]. - The $\mathrm{A} \cdot \mathrm{T}(\mathrm{wWC}) \leftrightarrow \mathrm{A} \cdot \mathrm{T}(w \perp W C), A \cdot T(w r W C) \leftrightarrow A \cdot T \mathrm{O} 2(\mathrm{w} \perp \mathrm{rWC}), \mathrm{A} \cdot \mathrm{T}(\mathrm{wH}) \leftrightarrow \mathrm{A} \cdot \mathrm{T}(w \perp H)$, $A \cdot T(w r H) \leftrightarrow A \cdot T \mathrm{O} 2(\mathrm{w} \perp \mathrm{rH})$ tautomeric transitions proceed through the stepwise proton transfer via the TSs as tight $\mathrm{A}+\cdot \mathrm{T}$ - ion pairs $(\Delta \mathrm{G}=20.76,23.06,19.00$ and $21.48 \mathrm{kcal} \cdot \mathrm{mol}-1$ in the continuum with $\varepsilon=1$ at $\mathrm{T}=298.15 \mathrm{~K})$, forming the conformationally-labile complexes $\mathrm{A} \cdot \mathrm{T}(w \perp W C), A \cdot T \mathrm{O} 2(\mathrm{w} \perp \mathrm{rWC}), \mathrm{A} \cdot \mathrm{T}(w \perp H)$ and $A \cdot \mathrm{TO} 2(\mathrm{w} \perp \mathrm{rH})$ [4]. Conclusions. These data enable to understand the physico-chemical mechanisms of the opening of the DNA base pairs and to describe in details the breathing of the DNA molecule. References. [1] Brovarets', O. O., Tsiupa, K. S., \& Hovorun, D. M. Front. Chem. 2018, 6:8, doi: 10.3389/ fchem.2018.00008. [2] Brovarets', O. O., Tsiupa, K. S., \& Hovorun, D. M. Sci. Repts. 2018, 8, 10371. [3] Brovarets', O. O., Tsiupa, K. S., \& Hovorun, D. M. Int. J. Quantum. Chem. 2018, 118, e25674. [4] Brovarets' O. O., Tsiupa K. S., Dinets A., Hovorun D. M. Front. Chem. 2018, 6:532; doi: 10.3389/fchem.2018.00532.
\end{abstract}




\title{
Study on characteristics of abdominal acute inflammation in mice to assess the therapeutic activity of human umbilical cord mses
}

\author{
P. Pikus, S. Rymar, N. Shuvalova, V. Kordium \\ Institute of Molecular Biology and Genetics of NAS of Ukraine
}

Background.Mesenchymal Stem Cells (MSCs) are the most promising candidates for regenerative medicine due to their unique properties. Today it is known that the therapeutic effect of transplanted MSCs primarily connected with their influence on inflammation, which accompanies almost all diseases. However, clinical trials have found that the effect of MSC transplantation is not always the same as expected because of their short term survival. The recent studies have shown on models of various diseases that preconditioning of MSCs changes their therapeutic potential.. Aim. The aim of this study was to evaluate the therapeutic effect of native and preconditioned HUCMSCs on sterile inflammation induced by the intraperitoneal injections of proteose peptone in mice. Methods. Experiments were performed on male BALB/c mice. Sterile inflammation was caused by intraperitoneal injection of $1 \mathrm{ml}$ of sterile $3 \%$ proteose-peptone solution. Control group of mice were injected with $1 \mathrm{ml}$ of saline solution. The development of inflammation was evaluated by the total number of cells and macrophages in peritoneal lavage fluid and via of macrophage phagocytic activity. MSCs were isolated from human umbilical cord using explants method. MSCs were preconditioned in the medium for 24 hours with $30 \mu \mathrm{mol} \mathrm{H} 2 \mathrm{O} 2$. In first series of experiments, mice were injected with different amounts native MSCs and preconditioned MSCs - H2O2 second passage $(2,5 \times 106 \mathrm{cells} / \mathrm{kg}, 0,8 \times 106$ cells $/ \mathrm{kg}, 0,4 \times 106 \mathrm{cells} / \mathrm{kg}$ of weight) $20 \mathrm{~min}$. after the injections of the proteose-peptone solution. During the second series of experiments a similar number of MSCs were injected $24 \mathrm{~h}$. after the injections of the proteose-peptone solution. Results. Injection of MSCs $20 \mathrm{~min}$. after the induction of sterile inflammation led to the disappearance of signs of inflammation in $4 \mathrm{~h}$. All used HUCMSCs concentrations have given the same effects. If animals were injected of native MSCs and MSCs- $\mathrm{H} 2 \mathrm{O} 2(2,5 \times 106 \mathrm{cell} / \mathrm{sg}) 24 \mathrm{~h}$. after induction of inflammation, then its signs disappeared after $4 \mathrm{~h}$. When mice were injected low doses of native MSCs and MSCs- H2O2 (0,4x106cells $/ \mathrm{kg}) 24 \mathrm{~h}$. after induction of inflammation, then its signs decreased by 2,8 times and 5 times, respectively. This indicates a significant difference in the therapeutic effect between native and preconditioned MSCs. Conclusions. The study showed that the use of a low doses of human umbilical cord MSCs allow to very clearly determine the difference in the therapeutic activity of MSCs. The factors found will be used when different MSCs preconditioning approaches. 


\title{
Novel conformationally-tautomeric diversity of the g.c DNA base: a quantum-mechanical study
}

\author{
O. Brovarets, Timothy A. Oliynyk, Dmytro M. Hovorun \\ Institute of Molecular Biology and Genetics of NAS of Ukraine
}

\begin{abstract}
Aim. To uncover novel conformers of the $G \cdot C$ DNA base pairs (asterisks denote rare tautomers), in particular, reverse Löwdin $\mathrm{G} \cdot C(\mathrm{rWC})$, Hoogsteen $(\mathrm{H}) \mathrm{G}^{\prime} \cdot C(\mathrm{H})$ and reverse Hoogsteen $\mathrm{G}^{\prime} \cdot C(\mathrm{rH})$ base pairs. Methods. MP2/aug-cc-pVDZ//B3LYP/6-311++G(d,p) level of quantummechanical theory in the continuum with $\varepsilon=1$ under normal conditions in combination with Bader's quantum theory of "Atoms in Molecules". Results. We have found that these structures are formed by means of the tautomerization process via proton transfer (PT) between the bases along the intermolecular H-bonds: $-\mathrm{G} \cdot C(\mathrm{rWC}) \leftrightarrow \mathrm{G}+\cdot \mathrm{C}-(\mathrm{rWC})$ (Gibbs free energy of activation $4.38 \mathrm{kcal} \cdot \mathrm{mol}-1) \leftrightarrow \mathrm{G} \cdot \mathrm{CO} 2(\mathrm{rWC})(3.64 \mathrm{kcal} \cdot \mathrm{mol}-1)$ and $G \cdot \mathrm{C}(r W C) \leftrightarrow G+\cdot C-$ $(r W C) \leftrightarrow G \mathrm{~N} 2 \cdot \mathrm{C}(\mathrm{rWC})(9.27 \mathrm{kcal} \cdot \mathrm{mol}-1)$ reactions occur via the two-stage sequential PT via the dynamically-unstable zwitterion-like $\mathrm{G}+\cdot \mathrm{C}-(\mathrm{rWC})$ intermediate, and transition states (TSs), one of which is joint; - G.C(rWC)↔G ${ }^{\prime} N 2 \cdot C(r w W C)(27.43 \mathrm{kcal} \cdot \mathrm{mol}-1), G^{\prime} \cdot \mathrm{C}(\mathrm{H}) \leftrightarrow G \mathrm{~N} 7 \cdot \mathrm{C}(\mathrm{H})$ $(4.01 \mathrm{kcal} \cdot \mathrm{mol}-1)$ and $\mathrm{G}^{\prime} \cdot C(\mathrm{rH}) \leftrightarrow \mathrm{G}^{* \prime} \mathrm{N} 7 \cdot \mathrm{C}(\mathrm{rH})(25.30 \mathrm{kcal} \cdot \mathrm{mol}-1)$ reactions occur through one-stage double PT. Also, we have conducted a detailed analisys of the evolution of main physico-chemical parameters along the intrinsic reaction coordinate by applying the author's unique methodology. Conclusions. The obtained data are useful in furthering our understanding of the conformational and tautomeric variability of the classical G.C DNA base pairs.
\end{abstract}




\title{
Comparative evaluation of the saturation efficiency of isolated grape buds with cryoprotectants
}

\author{
A. Prystalov, O. M. Bobrova, L. G. Kuleshova \\ Institute for Problems of Cryobiology and Cryomedicine of the NAS of Ukraine
}

\begin{abstract}
Aim: A comparative analysis of the efficiency of isolated grapes dormant buds saturation with plant vitrification solutions (PVS) by the vacuum infiltration vitrification (VIV) cryopreservation method and passive saturation method. Methods: Grapes dormant buds (Russian Concord variety) with a small site of wood $20-25 \mathrm{~mm}$ were isolated from an annual vine collected in the autumn-winter period. Isolated grape buds were placed in cryopreservation vials with cryopreservation medium PVS 2 (30\% glycerol, $15 \%$ ethylene glycol $15 \%$ DMSO, $0.4 \mathrm{M}$ sucrose), PVS 3 (44 \% glycerol, $44 \%$ sucrose) or PVS 4 (35\% glycerol, $20 \%$ ethylene glycol, $0.6 \mathrm{M}$ sucrose) prepared in the Murashige-Skoog culture medium. The two methods of saturation were compared: 1 . classical passive saturation (incubation in cryoprotective solutions at normal atmospheric pressure for $120 \mathrm{~min}$ ); 2. VIV (incubation for 30 minutes after a reduced pressure of 20-60 $\mathrm{mm} \mathrm{Hg}$ ). The saturation efficiency was estimated with the method of low temperature differential scanning calorimetry (DSC) by changing the temperatures of phase transitions, reducing of the enthalpy of water crystallization, and increasing the jump in heat absorption during glass transition. The samples were cooled down to $-196{ }^{\circ} \mathrm{C}$ by immersing into liquid nitrogen (an average cooling rate was $200 \mathrm{deg} / \mathrm{min}$ ), the thermograms were recorded at the heating rate of $0.5 \mathrm{deg} / \mathrm{min}$. Results: We have found that the reduced pressure in the tissues of the grapes dormant buds before immersion into a cryopreservation solution (30 min incubation) leads to a greater penetration of cryoprotective substances in the grapes buds tissue compared with the standard incubation procedure for 120 minutes. Thus, for example, on the DSC thermograms of the grapes buds saturated with the PVS 2 solution, using the VIV method, the heat absorption jump of glass transition is twice as high, the melting point is 4 degrees lower and the enthalpy of melting of the bound water is 2,9 times higher than in case of the classical saturation method. The results suggest an increase in the concentration of cryoprotective substances in the grapes buds and a significant decrease in the amount of free water that crystallizes during cooling. Since crystallization of ice is the main damaging factor in the cryopreservation of biological objects, the use of a modern method of saturation of grape buds can greatly increase their survival in cryopreservation. A long incubation period in cryopreservation solutions for classical saturation method can lead to the damage of the surface cells layer of grapes buds. Conclusions: the use of the VIV method to saturate isolated grapes dormant buds with PVS solutions makes it possible to reduce the incubation time by four times compared to passive saturation, while allowing for a higher concentration of cryoprotectants in the grapes dormant buds' tissues.
\end{abstract}




\title{
Oxidative stress caused by ozone as factor for changing index of sphericity of red blood cells at hypothermic storage
}

\author{
K. Holovina, Yu. S. Hovorova, I. F. Kovalenko, O. O. Shapkina, O. M. Bobrova \\ Institute for Problems of Cryobiology and Cryomedicine of the National Academy of Sciences of Ukraine
}

Background. Autotransfusions of ozonated blood or infusions of gaseous ozone into blood vessels and body cavities are effective in curing some pathological states, but the mechanism of ozone interaction with blood components is still unknown (Gornicki A., 2013). That is why, research of the ozone influence on the structural and functional parameters of blood components and particular erythrocytes, is an important aspect in modern science. It is known that warmed ovine erythrocytes in addition to their possible use in veterinary medicine are widely used in various studies such as immunological researches (Sparrow R.L. et al. 2015). Therefore, finding new and improving existing methods of storage is a perspective direction in cryobiology. For example, it has previously been shown that using the inducers of oxidative stress (particular, ozone) before freezing, increased efficiency of cryopreservation of red blood cells (Zinchenko V.D. et al., 2013). It is known that parameters of mechanical resistance are widely used to assess red blood cell state under conditions of stress factors (Shpakova N.M. et al., 2015). Therefore, the aim of this work was to study the effect of ozone treatment on erythrocyte sphericity index and mechanical stability at hypothermic storage. Methods. The washed human and ovine erythrocytes were ozonated (the dose of ozone was $120 \mu \mathrm{g} / \mathrm{ml}$ ) and resuspended in Olsver solution (in 1:1) or $5 \%$ mannitol solution on saline solution. Control and ozonized erythrocytes were stored in hypothermic conditions for 2 months. To study the distribution density of the eretrocytes sphericity index, we prepeared an experimental curve of osmotic fragility of erythrocytes. For this purpose, with the use of the low-angle scattering, the part of the preserved cells in the hypotonic $\mathrm{NaCl}$ solutions with concentrations of $0.9,0.8$, $0.7,0.6,0.5,0.4$ and $0.3 \%$ was determined. The curves of distribution of erythrocytes according to the spherical index were built using experimental curves of osmotic fragility based on the physical and mathematical model of hypotonic hemolysis in the solution of the non-penetrating substance. Results. Hypothermic storage of control and ozonated ovine erythrocytes for 2 months results in a shift of the density curve of its distribution by the sphericity index to the range of higher spherical indexes, and in the medium with mannitol, this shift is more distinct. After hypothermic storage of human red blood cells, the proportion of cells with low sphericity index increases sharply in the medium with mannitol as well as in the Olsver medium. Conclusions. The density of distribution of erythrocytes by the index of sphericity after hypothermic storage depends on the preservation medium. 


\title{
The effect of cytokine emap ii on the viability of glial cells in the primary culture derived from human glioma
}

\author{
I. Shuba ${ }^{1}$, V. Lylo ${ }^{2}$, O. Glavatskyi ${ }^{1}$, O. Kornelyuk ${ }^{2}$ \\ ${ }^{1}$ The State Institution "Romodanov Neurosurgery Institute, National Academy of Medical Sciences of Ukraine", \\ Kyiv, Ukraine \\ 2 Institute of Molecular Biology and Genetics of Natl. Acad. of Sci. of Ukraine, \\ Kyiv, Ukraine
}

Background: Malignant glial tumors of the brain are the most common form of oncological pathology of the brain with extremely unfavorable prognosis. The current strategy for treating this pathology is based on an integrated approach that includes surgical treatment, radiation and chemotherapy with drugs with an alkylating mechanism of action aimed at inhibiting DNA synthesis and mitotic activity of tumor cells, but these drugs have a rather high overall toxicity. Current efforts to improve GBM treatment include the addition of novel-targeted agents to the standard care regimen. One of the most promising approaches is the use of drugs with antiangiogenic properties, which include endothelial monocyte-activating polypeptide-II (EMAP-II). Previous studies have shown that EMAP-II stimulates apoptosis in tumor cells, is still relevant in studies of effects on gliomas, since malignant gliomas are characterized by pronounced vascularization associated with the production of prohangio-genic factors. The aim: To compare the cytotostatic and cytotoxic effects of the cytokine EMAP II on the primary culture of human glioma cells, as well as to detect doses of the drug causing cell death. Methods: Primary cells are extracted directly from the tissue and processed to establish them under culture conditions. Cultivation was carried out in a medium (DMEM) with the addition of $10 \%$ embryonal veal serum (Sigma, USA) in a CO2 incubator (ES-160 "Nüve", Turkey) under standard conditions ( $\mathrm{t}=370 \mathrm{C}, 5 \% \mathrm{CO} 2$ ). The recombinant EMAP II polypeptide was obtained in the Department of Protein Engineering and Bioinformatics, Institute of Molecular Biology and Genetics of the National Academy of Sciences of Ukraine, using genetic engineering methods. The viability of cells was determined by the inclusion of $4 \%$ trypan blue, counting the number of cells was performed using a light microscope and a camera Goryaev. Trypan blue dye selectively paints dead cells, counting the total number of cells and the percentage of live and dead cells after their treatment with drugs allows to evaluate its cytostatic and cytotoxic effects. Results: According to the literature, EMAP II studies were conducted on the U251 cell line, in our work, research was conducted on primary cell culture. The viability of the glomerular cells was determined after 24 hours. Incubation with EMAP II protein formulations in the concentration range from $1 \mathrm{nM}$ to $1024 \mathrm{nM}$. It has been shown that EMAP II exhibits dose-dependent cytotoxic properties with a maximum in the range of concentrations from $4 \mathrm{nM}$ to $64 \mathrm{nM}$. Conclusion: The EMAP II polypeptide is a promising compound for further research on anti-tumor activity in the treatment of brain's gliomas. 


\title{
Effect of PT(II) and PD(II) cisplatin analogues and chitosan on DNA-dependent processes
}

\author{
O. Sorokin ${ }^{1}$, H. Repich ${ }^{2}$, A. Likhanov ${ }^{3}$, T. Shyryna ${ }^{1}$, L. Palchykovska ${ }^{1}$ \\ ${ }^{1}$ Institute of Molecular Biology and Genetics NAS of Ukraine \\ ${ }^{2}$ V. I. Vernadskii Institute of General and Inorganic Chemistry NAS of Ukraine \\ ${ }^{3}$ Institute for evolutionary ecology NAS of Ukraine
}

Background: Modern experimental biology and computational technologies, allow understanding the important mechanisms of diseases development and drug actions. Researches in these fields resulted in a new concept of drug design, polypharmacology. It focuses on developing single drugs with multiple targets. It became increasingly valuable in anticancer, antimicrobial and antiviral drug design. Aim of the present work was to study the interaction with DNA and influence on some DNA-dependent processes of newly synthetized and natural compounds as well as to investigate their cytostatic/cytotoxic effect and antimicrobial properties. 17 novel cisplatin analogues $(\mathrm{Pd} 2+$ and $\mathrm{Pt} 2+$ mononuclear $\pi$-coordination complexes with verious ligands) and natural different molecular weight chitosan were the objects of our study. Results. It was shown that cisplatin analogues have ability to bind with linear and plasmid form of pTZ19R1 DNA, inhibit T7 phage transcription $\left(\mathrm{IC}_{50}\right.$ from $7 \mu \mathrm{M}$ to $\left.91,67 \mu \mathrm{M}\right)$, inhibit polymerase chain reaction (PCR) $\left(\mathrm{IC}_{50}\right.$ from $1,56 \mu \mathrm{M}$ to $\left.14,2 \mu \mathrm{M}\right)$ and influence on restriction enzymes using in vitro models. The restriction of pTZ19R1 DNA by endonucleases BamHI and HindIII was considerably inhibited by two Pd(II) complexes, that proved the importance of metal ions in coordination compounds. Four complexes possessed pronounced proapoptotic and cytotoxic activity with $\mathrm{IC}_{50}$ index in range of $1-100 \mu \mathrm{M}$ on HeLa cells. The high affinity of studied compounds to DNA correlated with observed cytostatic and proapoptotic levels and indirectly indicated their complicated antiproliferative mechanism of action due to damage of cell genetic apparatus. In recent years, environmental-friendly means have been developed for combating plant diseases as alternative to chemical pesticides on the base of natural compounds such as chitosan. In our work we used high molecular weight (HMW) chitosan from the insect puparia Hermetia illucens and low molecular weight (LMW) chitosan from the Agaricus bisporus fruit bodies. It was demonstrated that at chitosan concentrations in range of 50-1.5 $\mu \mathrm{g}$ / $\mathrm{ml}$ there was formation of the chitosan-DNA complexes, which displayed a lower mobility in agarose gel as compared to control DNA. The in vitro testing demonstrated the almost complete inhibition of the RNA synthesis by HMW chitosan in the concentration range of $50-6.25 \mu \mathrm{g}$ / $\mathrm{ml}$. The inhibitory effect of the LMW chitosan was less effective. The study of the effect of LMW and HMW chitosans on the PCR amplification showed that both samples reliably suppressed the synthesis of DNA fragments. Conclusion. The results of present study showed high affinity of all tested compounds to the linear and plasmid forms of DNA and inhibition of the activity of replication, transcription and restriction enzymes that use DNA as a substrate. 